\title{
EXACT QUASI-CLASSICAL ASYMPTOTICBEYOND MASLOV CANONICAL OPERATOR AND QUANTUM JUMPS NATURE
}

\author{
J. Foukzon ${ }^{1}$, A.A.Potapov ${ }^{2}$, S.A. Podosenov ${ }^{3}$ \\ ${ }^{1}$ Israel Institute of Technology. \\ ${ }^{2}$ IRE RAS, \\ ${ }^{3}$ All-Russian Scientific-Research Institute
}

\begin{abstract}
:
Exact quasi-classical asymptotic beyondWKB-theory and beyondMaslov canonical operatorto the Colombeau solutions of then-dimensional Schrodinger equationis presented. Quantum jumps nature is considered successfully. We pointed out that an explanation ofquantum jumps can be found to result from Colombeausolutions of the Schrödinger equation alone without additional postulates.
\end{abstract}

\section{Keywords:}

Quantum jumps, quantum measurements theory, quantum averages, limiting quantum trajectory, Schrodinger equation,Stochastic quantum jump equation,Colombeau solution,Feynman path integral,Maslov canonical operator, Feynman-Colombeau propagator.

\section{Introduction}

A number of experiments on trapped single ions or atoms have been performed in recent years $[1,2,3,4]$. Monitoring the intensity of scattered laser light off of such systems has shown abrupt changes that have been cited as evidence of "quantum jumps" between states of the scattered ion or atom . The existence of such jumps was required by Bohr in his theory of the atom.Bohr's quantum jumps between atomic states [5] were thefirst form of quantum dynamics to be postulated.He assumed that an atom remained in an atomic eigen state until it made an instantaneous jump to another state with the emission or absorption of a photon. Since these jumps do not appear to occur in solutions of the Schrodinger equation, something similar to Bohr's idea has been added as an extra postulate in modern quantum mechanics.

Stochastic quantum jump equations [6], [7],[8]were introduced as a tool for simulating the dynamics of a dissipative system with a large Hilbert space and their links with quantum measurement theorwere also noted[9],[10],[11],[12],[13].This measurement interpretation is generally known as quantum trajectory theory [14].By adding filter cavities as part of the apparatus, even the quantum jumps in the dressed state model can be interpreted as approximations to measurement-induced jumps [15].

The question arises whether an explanation of these jumps can be found to result from an Colombeau solution [16]-[18] $\left(\Psi_{\varepsilon}(x, t ; \hbar)\right)_{\varepsilon}$ of the Schrödinger equation alone without additional postulates. We found exact quasi-classical asymptotic of the quantum averages with position variable with localized initial data. 
International Journal of Recent advances in Physics (IJRAP) Vol.3, No.4, November 2014

$\left(\left\langle i, t, x_{0} ; \hbar, \varepsilon\right\rangle\right)_{\varepsilon}=\left(\int x_{i}\left|\Psi_{\varepsilon}\left(x, t, x_{0} ; \hbar\right)\right|^{2} d x\right)_{\varepsilon^{\prime}}, \varepsilon \in(0,1], x, x_{0} \in \mathbb{R}^{d}, i=1, \ldots, d$,

i.e. we found the limiting Colombeau quantum averages (limiting Colombeau quantum trajectories) such that[18]:

$$
\begin{gathered}
\left(\left\langle i, t, x_{0} ; \varepsilon\right\rangle\right)_{\varepsilon}=\left(\underline{\lim }_{\hbar \rightarrow 0}\left\langle i, t, x_{0} ; \hbar, \varepsilon\right\rangle\right)_{\varepsilon}= \\
=\left(\underline{\lim }_{\hbar \rightarrow 0} \int x_{i}\left|\Psi_{\varepsilon}\left(x, t, x_{0} ; \hbar\right)\right|^{2} d x\right)_{\varepsilon^{\prime}}, \varepsilon \in(0,1], x \in \mathbb{R}^{d}, i=1, \ldots, d, t \in[0, T](1.2)
\end{gathered}
$$

And limiting quantum trajectories $\boldsymbol{q}\left(t, x_{0}\right)=\left\{q_{1}\left(t, x_{0}\right), \ldots, q_{d}\left(t, x_{0}\right)\right\} \in \mathbb{R}^{d}, t \in[0, T]$ such that $q_{i}\left(t, x_{0}\right)=\left\langle i, t, x_{0}\right\rangle=\lim _{\varepsilon \rightarrow 0} \lim _{\hbar \rightarrow 0}\left\langle i, t, x_{0} ; \hbar, \varepsilon\right\rangle=$

$=\lim _{\varepsilon \rightarrow 0} \lim _{\hbar \rightarrow 0} \int x_{i}\left|\Psi_{\varepsilon}\left(x, t, x_{0} ; \hbar\right)\right|^{2} d x, x \in \mathbb{R}^{d}, i=1, \ldots, d(1.3)$

if limit in LHS of Eq.(1.3) exist.

The physical interpretation of these asymptotic given below, shows that the answer is "yes" for the limiting quantum trajectories with localized initial data.

Note that an axiom of quantum measurement is:if the particle is in some state $\left|\Psi_{t, \varepsilon}\right\rangle$ thatthe probability $P(x, \delta x)$ of getting a result $x \in \mathbb{R}^{d}$ at instant $t$ with an accuracy of $\|\delta x\| \ll 1$ will be given by

$P_{t}\left(x, \delta x, x_{0} ; \hbar, \varepsilon\right)=\int_{\left\|x-x^{\prime}\right\| \leq\|\delta x\|}\left|\left\langle x^{\prime} \mid \Psi_{t, \varepsilon}\right\rangle\right|^{2} d x^{\prime}=\int_{\left\|x-x^{\prime}\right\| \leq\|\delta x\|}\left|\Psi_{\varepsilon}\left(x^{\prime}, t, x_{0} ; \hbar\right)\right|^{2} d x^{\prime} .(1.4)$

We rewrite now Eq.(1.4) of the form

$P_{t}\left(x, \delta x, x_{0} ; \hbar, \varepsilon\right)=\frac{1}{(2 \pi)^{d / 2}\|\delta x\|^{d}} \int_{\mathbb{R}^{d}}\left|\Psi_{\varepsilon}\left(x^{\prime}, t, x_{0} ; \hbar\right)\right|^{2} \exp \left[-\frac{\left\|x-x^{\prime}\right\|^{2}}{\|\delta x\|^{2}}\right] d x^{\prime} \cdot(1.5)$

We define well ocalized limiting quantum trajectories

$\boldsymbol{q}(t)=\boldsymbol{q}\left(t, x_{0}\right)=\left\{q_{1}\left(t, x_{0}\right), \ldots, q_{d}\left(t, x_{0}\right)\right\} \in \mathbb{R}^{d}$,

$\boldsymbol{q}\left(0, x_{0}\right)=x_{0}, t \in[0, T]$ such that:

$\left(\underline{\lim }_{\|\delta \boldsymbol{q}(t)\| \rightarrow 0} \underline{\lim }_{\hbar \rightarrow 0} P_{t}\left(\boldsymbol{q}(t), \delta \boldsymbol{q}(t), x_{0} ; \hbar, \varepsilon\right)\right)_{\varepsilon}=1(1.6)$

And well localized limiting quantum trajectories $\boldsymbol{q}(t)=\boldsymbol{q}\left(t, x_{0}\right)=\left\{q_{1}\left(t, x_{0}\right), \ldots, q_{d}\left(t, x_{0}\right)\right\} \in \mathbb{R}^{d}$, $\boldsymbol{q}\left(0, x_{0}\right)=x_{0}, t \in[0, T]$ such that:

$\lim _{\varepsilon \rightarrow 0} \lim _{\|\delta \boldsymbol{q}(t)\| \rightarrow 0} \lim _{\hbar \rightarrow 0} P_{t}\left(\boldsymbol{q}(t), \delta \boldsymbol{q}(t), x_{0} ; \hbar, \varepsilon\right)=1(1.7)$

if limit in LHS of Eq.(1.7) exist. 


\section{Colombeau solutions of the Schrödinger equation and correspondingpath integral representation}

Let $\mathbf{H}$ be a complex infinite dimensional separable Hilbert space, with inner product $\langle\cdot, \cdot\rangle$ and norm $|\cdot|$.

Let us considerSchrödinger equation:

$-i \hbar\left(\frac{\partial \Psi(t)}{\partial t}\right)+\widehat{H}(t) \Psi(t)=0, \Psi(0)=\Psi_{0}(x),(2.1)$

$H(t)=-\left(\frac{\hbar^{2}}{2 m}\right) \Delta+V(x, t) \cdot(2 \cdot 2)$

Hereoperator $H(t): \mathbb{R} \times \mathbf{H} \rightarrow \mathbf{H}$ is essentially self-adjoint, $\widehat{H}(t)$ is the closure of $H(t)$.

Theorem 2.1. [19],[20].Assume that:(1) $\Psi_{0}(\mathrm{x}) \in L_{2}\left(\mathbb{R}^{d}\right),(2) \quad V(x, t)$ is continuous and $\left.\sup _{x \in \mathbb{R}^{d}, t \in[0, T]}|V(x, t)|\right)<+\infty$. Then corresponding solution of the Schrödinger equation (2.1)-(2.2)

exist and can be represented via formulae

$\Psi(t, x)=\lim _{n \rightarrow \infty}\left(\frac{n m}{4 \pi i t \hbar}\right)^{d(n+1) / 2} \int_{\mathbb{R}^{d}} \ldots \int_{\mathbb{R}^{d}} d x_{0} d x_{1} \ldots d x_{n} \Psi_{0}\left(x_{0}\right) \exp \left[\frac{i}{\hbar} S\left(x_{0}, x_{1}, \ldots, x_{n}, x_{n+1} ; t\right)\right],(2.3)$

where we have set $x_{n+1}=x$ and

$S\left(x_{0}, x_{1}, \ldots, x_{n}, x_{n+1} ; t\right)=\sum_{i=1}^{n}\left[\frac{m}{4} \frac{\left|x_{i+1}-x_{i}\right|^{2}}{(t / n)^{2}}-\mathrm{V}\left(x_{i+1}, t_{i}\right)\right],(2.4)$

where $t_{i}=\frac{i t}{n}$. Let $q_{n}(t)$ be a trajectory; that is, a function from $[0, t]$ to $\mathbb{R}^{d}$ with $q_{n}(0)=x_{0}$ and set $q_{n}\left(t_{i}\right)=$ $x_{i}, i=1, \ldots, n+1$.We rewrite Eq.(2.3) for a future application symbolically for short of the following form

$\Psi(t, x)=\lim _{n \rightarrow \infty} \int_{q_{n}(t)=x} D\left[q_{n}(t)\right] \Psi_{0}\left(q_{n}(0)\right) \exp \left[\frac{i}{\hbar} S\left(q_{n}(t), x ; t\right)\right],(2.5)$

where we have set(i) $S\left(q_{n}(t), x ; t\right)=S\left(x_{0}, x_{1}, \ldots, x_{n}, x_{n+1} ; t\right)$ and (ii) $D\left[q_{n}(t)\right]$ that is, a

$D\left[q_{n}(t)\right]=\left(\frac{n m}{4 \pi i t \hbar}\right)^{d(n+1) / 2} \prod_{j=0}^{n} d x_{j} \cdot(2.6)$

Trotter and Kato well known classical results give a precise meaning to the Feynmanintegral when the potential $V(x, t)$ is sufficiently regular[18]-[19]. However if potential $V(x, t)$ is a non-regular this is well known problem to represent solution of the Schrödinger equation (2.1)-(2.2) via formulae(2.3), see [19].

We avoided this difficulty using contemporaryColombeauframework[16]-[18]. Using replacement $x_{i} \rightarrow$ $\frac{x_{i}}{1+\varepsilon^{2 k}|x|^{2 k}}, \varepsilon \in(0,1], k \geq 1$ we obtain from potential $V(x, t)$ regularized potential $V_{\varepsilon}(x, t), \varepsilon \in(0,1]$, such that $V_{\varepsilon=0}(x, t)=V(x, t)$ and

(i) $\quad\left(V_{\varepsilon}(x, t)\right)_{\varepsilon} \in G\left(\mathbb{R}^{d}\right)$,

(ii) $\left.\quad \sup _{x \in \mathbb{R}^{d}, t \in[0, T]}\left|V_{\varepsilon}(x, t)\right|\right)<+\infty, \varepsilon \in(0,1] .(2.7)$ 
International Journal of Recent advances in Physics (IJRAP) Vol.3, No.4, November 2014

Here $G\left(\mathbb{R}^{d}\right)$ isColombeau algebra of Colombeau generalized functions [16]-[18].

Finally we obtainregularizedSchrödinger equation of Colombeau form[16]-[18]:

$-i \hbar\left(\frac{\partial \Psi_{\varepsilon}(t)}{\partial t}\right)_{\varepsilon}+\left(\widehat{H}_{\varepsilon}(t) \Psi_{\varepsilon}(t)\right)_{\varepsilon}=0,\left(\Psi_{\varepsilon}(0)\right)_{\varepsilon}=\Psi_{0}(x),(2.8)$

$H_{\varepsilon}(t)=-\left(\frac{\hbar^{2}}{2 m}\right) \Delta+V_{\varepsilon}(x, t) \cdot(2.9)$

Using the inequality (2.7) Theorem 2.1 asserts again that corresponding solution of the Schrödinger equation (2.8)-(2.9)exist and can be represented via formulae [18]:

$$
\begin{gathered}
\left(\Psi_{\varepsilon}(t, x)\right)_{\varepsilon}= \\
\left(\lim _{n \rightarrow \infty}\left(\frac{n m}{4 \pi i t \hbar}\right)^{d(n+1) / 2} \int_{\mathbb{R}^{d}} \ldots \int_{\mathbb{R}^{d}} d x_{0} d x_{1} \ldots d x_{n} \Psi_{0}\left(x_{0}\right) \exp \left[\frac{i}{\hbar} S_{\varepsilon}\left(x_{0}, x_{1}, \ldots, x_{n}, x_{n+1} ; t\right)\right]\right)_{\varepsilon}
\end{gathered}
$$

where we have set $x_{n+1}=x$ and

$S_{\varepsilon}\left(x_{0}, x_{1}, \ldots, x_{n}, x_{n+1} ; t\right)=\sum_{i=1}^{n}\left[\frac{m}{4} \frac{\left|x_{i+1}-x_{i}\right|^{2}}{(t / n)^{2}}-V_{\varepsilon}\left(x_{i+1}, t_{i}\right)\right],(2.11)$

where we have set $t_{i}=\frac{i t}{n}$.

We rewrite Eq.(2.10) for a future application symbolically of the following form

$$
\left(\Psi_{\varepsilon}(t, x)\right)_{\varepsilon}=\left(\lim _{n \rightarrow \infty} \int_{q_{n}(t)=x} D\left[q_{n}(t)\right] \Psi_{0}\left(q_{n}(0)\right) \exp \left[\frac{i}{\hbar} S_{\varepsilon}\left(q_{n}(t) ; t\right)\right]\right)_{\varepsilon}
$$

Or of the following form

$$
\left(\Psi_{\varepsilon}(t, x)\right)_{\varepsilon}=\left(\lim _{n \rightarrow \infty} \Psi_{\varepsilon, \mathrm{n}}(t, x)\right)_{\varepsilon}=\left(\lim _{n \rightarrow \infty} \int_{q(t)=x} D_{n}[q(t)] \Psi_{0}\left(q_{n}(0)\right) \exp \left[\frac{i}{\hbar} S_{\varepsilon}(\dot{q}(t), q(t) ; t)\right]\right)_{\varepsilon}
$$

For the limit in RHS of (2.12) and (2.13) we will be used canonical path integral notation

$$
\left(\Psi_{\varepsilon}(t, x)\right)_{\varepsilon}=\left(\int_{q(t)=x} D[q(t)] \Psi_{0}(q(0)) \exp \left[\frac{i}{\hbar} S_{\varepsilon}(\dot{q}(t), q(t))\right]\right)_{\varepsilon},(2.1
$$

where $S_{\varepsilon}(\dot{q}(t), q(t))=\int_{0}^{t}\left[\frac{m}{4} \dot{q}^{2}(s)-V_{\varepsilon}(q(s), s)\right] d s$.

Substitutionn $=8 k+7$ into RHS of the Eq.(2.10) gives

$$
\begin{gathered}
\left(\Psi_{\varepsilon}(t, x)\right)_{\varepsilon}= \\
\left(\lim _{k \rightarrow \infty}\left(\frac{(8 k+7) m}{4 \pi t \hbar}\right)^{d(4 k+4)} \int_{\mathbb{R}^{d}} \ldots \int_{\mathbb{R}^{d}} d x_{0} d x_{1} \ldots d x_{8 k+7} \Psi_{0}\left(x_{0}\right) \exp \left[\frac{i}{\hbar} S_{\varepsilon}\left(x_{0}, x_{1}, \ldots, x_{8 k+7}, x_{8 k+8} ; t\right)\right]\right)_{\varepsilon} .(2 .)
\end{gathered}
$$

We rewrite Eq.(2.15) for a future application symbolically of the following form 
International Journal of Recent advances in Physics (IJRAP) Vol.3, No.4, November 2014

$$
\left(\Psi_{\varepsilon}(t, x)\right)_{\varepsilon}=\left(\lim _{n \rightarrow \infty} \int_{q_{n}(t)=x} D^{+}\left[q_{n}(t)\right] \Psi_{0}\left(q_{n}(0)\right) \exp \left[\frac{i}{\hbar} S_{\varepsilon}\left(q_{n}(t) ; t\right)\right]\right)_{\varepsilon}^{\prime}
$$

or of the following form

$$
\left(\Psi_{\varepsilon}(t, x)\right)_{\varepsilon}=\left(\lim _{n \rightarrow \infty} \int_{q(t)=x} D_{n}^{+}[q(t)] \Psi_{0}(q(0)) \exp \left[\frac{i}{\hbar} S_{\varepsilon}(\dot{q}(t), q(t) ; t)\right]\right)_{\varepsilon}
$$

For the limit in RHS of (2.16) and(2.17) we will be used following path integral notation

$$
\left(\Psi_{\varepsilon}(t, x)\right)_{\varepsilon}=\left(\int_{q(t)=x} D^{+}[q(t)] \Psi_{0}(q(0)) \exp \left[\frac{i}{\hbar} S_{\varepsilon}(\dot{q}(t), q(t))\right]\right)_{\varepsilon} .(2.1
$$

Let us consider now regularized oscillatory integral

$$
\left(\mathrm{J}_{\varepsilon, n}(t ; \hbar)\right)_{\varepsilon}=\left(\int_{\mathbb{R}^{d}} \ldots \int_{\mathbb{R}^{d}} d x_{0} d x_{1} \ldots d x_{n} f\left(x_{0}, x_{1}, \ldots, x_{n}\right) \Psi_{0}\left(x_{0}\right) \exp \left[\frac{i}{\hbar} S_{\varepsilon}\left(x_{0}, x_{1}, \ldots, x_{n} ; t\right)\right]\right)_{\varepsilon} \text {. }
$$

Lemma2.1.(Localization Principle [25]-[26])Let $\Omega$ be a domain in $\mathbb{R}^{d \times n}$ and $f \in C_{0}^{\infty}(\Omega)$ be a smooth functionof compact support, $S_{\varepsilon} \in C^{\infty}(\Omega), \varepsilon \in(0,1]$ be a real valued smooth function withoutstationary points in $\operatorname{supp}(f)$, i.e. $\partial_{x} S_{\varepsilon}(x) \neq 0$ for $x \in \Omega$. Let $L$ be a differential operator

$L(f)=-\sum_{i=1}^{n} \frac{\partial}{\partial x_{i}}\left(\left|S_{\varepsilon, x}^{\prime}(x)\right|^{-2} \frac{\partial S_{\varepsilon}}{\partial x_{i}} f\right)$.

Then

$\left(\left|\mathrm{J}_{\varepsilon, n}(t ; \hbar)\right|\right)_{\varepsilon} \leq \hbar \int_{\Omega} L(f(x)) d x . \forall n, m \in \mathbb{N}, \forall \hbar \leq 1$ thereexist $c_{m}$ such that

$$
\left(\mathrm{J}_{\varepsilon, n}(t ; \hbar)\right)_{\varepsilon} \leq c_{m} \hbar^{m}\|f\|,\|f\|=\sup _{x \in \Omega} \sum_{|\alpha| \leq m}\left|D^{\alpha} f\right|
$$

Lemma2.2. (Generalized Localization Principle) Let $\Omega_{n}$ be a domain in $\mathbb{R}^{d \times n}$ and $f_{n} \in C_{0}^{\infty}\left(\Omega_{n}\right)$ be a real valued smooth function without stationary points in $\operatorname{supp}(f)$, i.e. $\partial_{x} S_{\varepsilon}(x) \neq 0$ for $x \in \Omega_{n}$ and let $\left(\wp_{\varepsilon}(t ; \hbar)\right)_{\varepsilon}$ be infinite sequencen $\in \mathbb{N}$ :

$$
\begin{gathered}
\left(\wp_{\varepsilon, n}(t ; \hbar)\right)_{\varepsilon}= \\
\left(\left(\frac{n m}{4 \pi i t \hbar}\right)^{d(n+1) / 2} \int_{\Omega_{n}} d x_{0} d x_{1} \ldots d x_{n} f_{n}\left(x_{0}, x_{1}, \ldots, x_{n}\right) \Psi_{0}\left(x_{0}\right) \exp \left[\frac{i}{\hbar} S_{\varepsilon}\left(x_{0}, x_{1}, \ldots, x_{n} ; t\right)\right]\right)_{\varepsilon} .
\end{gathered}
$$

Then there exist infinite sequence $\left\{\hbar_{k}\right\}_{k \in \mathbb{N}}, \lim _{k \rightarrow \infty} \hbar_{k}=0$ such that

$$
\left(\lim _{\substack{k \rightarrow \infty \\ k \rightarrow \infty}} \wp_{\varepsilon, n}\left(t ; \hbar_{k}\right)\right)_{\varepsilon}=0 .
$$

Proof.Equality (2.23) immediately follows from (2.21).

Remark2.1.FromLemma2.2follows thatstationary phase approximationis not a valid asymptotic approximation in the limit $\hbar \rightarrow 0$ for a path-integral (2.14) and (2.18). 


\section{Exactquasi-classical asymptotic beyond Maslov canonical operator}

Theorem3.1.Letus considerCauchy problem (2.8) with initial data $\Psi_{0}(x)$ is givenvia formula

$\Psi_{0}(x)=\frac{\eta^{d / 4}}{(2 \pi)^{d / 4} \hbar^{d / 4}} \exp \left[-\frac{\eta\left(x-x_{0}\right)^{2}}{2 \hbar}\right]$

where $0<\hbar \ll \eta \ll 1$ and $x^{2}=\langle x, x\rangle$.

(1) We assume now that: (i) $\left(V_{\varepsilon}(x, t)\right)_{\varepsilon} \in G\left(\mathbb{R}^{d}\right)$, (ii) $V_{\varepsilon=0}(x, t)=V(x, t): \mathbb{R}_{+} \times \mathbb{R}^{d} \rightarrow \mathbb{R}$ and

(iii) $\forall t \in \mathbb{R}_{+}$function $V(x, t)$ is a polynomial on variable $x=\left(x_{1}, \ldots, x_{d}\right)$, i.e.

$V(x, t)=\sum_{\|\alpha\| \leq m} g_{\alpha}(t) x^{\alpha}, \alpha=\left(i_{1}, \ldots, i_{d}\right), x^{\alpha}=x_{1}^{i_{1}} \times \ldots \times x_{d}^{i_{d}},\|\alpha\|=\sum_{r=1}^{d} i_{r}(3.2)$

(2) Let $u(\tau, t, \lambda, x, y)=\left(u_{1}(\tau, t, \lambda, x, y), \ldots, u_{d}(\tau, t, \lambda, x, y)\right)$ be the solution of the boundary problem

$\frac{\partial^{2} u^{\mathrm{T}}(\tau, t, \lambda, x, y)}{\partial \tau^{2}}=\operatorname{Hess}[V(\lambda, \tau)] u^{\mathrm{T}}(\tau, t, \lambda, x, y)+\left[V^{\prime}(\lambda, \tau)\right]^{\mathrm{T}},(3.3)$

$u(0, t, \lambda, x, y)=y, u(t, t, \lambda, x, y)=x .(3.4)$

Here $\lambda=\left(\lambda_{1}, \ldots, \lambda_{d}\right) \in \mathbb{R}^{d}, u^{\mathrm{T}}(\tau, t, \lambda, x, y)=\left(u_{1}(\tau, t, \lambda, x, y), \ldots, u_{d}(\tau, t, \lambda, x, y)\right)^{\mathrm{T}}$,

$V^{\prime}(\lambda, \tau)=\left(\left[\frac{\partial V(x, t)}{\partial x_{1}}\right]_{x=\lambda}, \ldots,\left[\frac{\partial V(x, t)}{\partial x_{d}}\right]_{x=\lambda}\right) \operatorname{andHess}[V(\lambda, \tau)]=\left[\frac{\partial^{2} V(x, t)}{\partial x_{i} \partial x_{j}}\right]_{x=\lambda}$

(3) $\operatorname{LetS}(t, \lambda, x, y)$ be the master action givenvia formula

$\mathrm{S}(t, \lambda, x, y)=\int_{0}^{t} L(\dot{u}(\tau, t, \lambda, x, y), u(\tau, t, \lambda, x, y), \tau) d \tau,(3.6)$

Where master LagrangianL $(\dot{u}, u, \tau)$ are

$$
\begin{aligned}
& L(\dot{u}, u, \tau)=\frac{m}{2} \dot{u}^{2}(\tau, t, \lambda, x, y)-\hat{V}(u(\tau, t, \lambda, x, y), \tau), \dot{u}=\left(\frac{\partial u_{1}}{\partial \tau}, \ldots, \frac{\partial u_{d}}{\partial \tau}\right), \dot{u}^{2}=\langle\dot{u}, \dot{u}\rangle,(3.7) \\
& \hat{V}((\tau, t, \lambda, x, y), \tau)=u(\tau, t, \lambda, x, y) \operatorname{Hess}[V(\lambda, \tau)] u^{\mathrm{T}}(\tau, t, \lambda, x, y)+V^{\prime}(\lambda, \tau) u^{\mathrm{T}}(\tau, t, \lambda, x, y) .
\end{aligned}
$$

Let $y_{c r}=y_{c r}(t, \lambda, x) \in \mathbb{R}^{d}$ be solution of the linear system of the algebraic equations

$\left[\frac{\partial \mathrm{S}(t, \lambda, x, y)}{\partial y_{i}}\right]_{y=y_{c r}}=0, i=1, \ldots, d$

(4) Let $\hat{x}=\hat{x}\left(t, \lambda, x_{0}\right) \in \mathbb{R}^{d}$ be solution of the linear system of the algebraic equations 
$y_{c r}(t, \lambda, \hat{x})+\lambda-x_{0}=0$.

Assume that: for a given values of the parameters $t, \lambda, x_{0}$ the point $\hat{x}=\hat{x}\left(t, \lambda, x_{0}\right)$ is not a focal point on a corresponding trajectory is given by corresponding solution of the boundary problem (3.3). Then forthe limiting quantum averagegiven via formulae (1.1) the inequalities is satisfied:

$$
\begin{gathered}
\underline{\lim }_{\substack{\hbar \rightarrow 0 \\
\varepsilon \rightarrow 0}}\left|\left\langle i, t, x_{0} ; \hbar\right\rangle-\lambda_{i}\right| \leq \\
\leq 2\left[\left|\operatorname{det} S_{y_{c r} y_{c r}}\left(t, \lambda, \hat{x}\left(t, \lambda, x_{0}\right), y_{c r}\left(t, \lambda, \hat{x}\left(t, \lambda, x_{0}\right)\right)\right)\right|\right]^{-1}\left|\hat{x}_{i}\left(t, \lambda, x_{0}\right)\right|, i=1, \ldots, d .(3.11)
\end{gathered}
$$

Thus one can to calculate the limiting quantum trajectory corresponding to potentialV $(x, t)$ by using transcendental masterequation

$$
\hat{x}_{i}\left(t, \lambda, x_{0}\right)=0, i=1, \ldots, d .(3.12)
$$

Proof. From inequality (A.15) and Theorem A1, using inequalities(A.53.a) and (A.53.b) we obtain

$\underline{\lim }_{\substack{\sigma \rightarrow 0 \\ \sigma \rightarrow 0}} \underline{\hbar \rightarrow 0}\left|\left\langle\hat{x}_{i}, T ; \sigma, l, \lambda, \varepsilon\right\rangle-\lambda_{i}\right| \leq \lim _{\hbar \rightarrow 0}\left[\mathcal{R}_{1}(T, \lambda)+\mathcal{R}_{2}(T, \lambda)\right], i=1, \ldots, d,(3.13)$

where

$$
\begin{aligned}
& \mathcal{R}_{1}(T, \lambda)=\int d x\left\{\int_{q(T)=x} D^{+}[q(t)] \Psi(q(0))\left[\left|q_{i}(T)\right|\right]^{\frac{1}{2}} \cos \left[\frac{1}{\hbar} \boldsymbol{S}_{1}(\dot{q}, q, \lambda, T)\right]\right\}^{2}, \\
& \mathcal{R}_{2}(T, \lambda)=\int d x\left\{\int_{q(T)=x} D^{+}[q(t)] \Psi(q(0))\left[\left|q_{i}(T)\right|\right]^{\frac{1}{2}} \sin \left[\frac{1}{\hbar} \boldsymbol{S}_{1}(\dot{q}, q, \lambda, T)\right]\right\}^{2} .
\end{aligned}
$$

We note that

$\mathcal{R}_{1}(T, \lambda)=\int d x\left[\breve{\mathcal{R}}_{1}(x, T, \lambda)\right]^{2}, \mathcal{R}_{2}(T, \lambda)=\int d x\left[\breve{\mathcal{R}}_{2}(x, T, \lambda)\right]^{2}$,

where

$$
\begin{gathered}
\breve{\mathcal{R}}_{1}(x, T, \lambda)=\int_{q(T)=x} D^{+}[q(t)] \Psi(q(0))\left[\left|q_{i}(T)\right|\right]^{\frac{1}{2}} \cos \left[\frac{1}{\hbar} \boldsymbol{S}_{1}(\dot{q}, q, \lambda, T)\right]= \\
=\int d y \int_{\substack{q(T)=x \\
q(0)=y}} D^{+}[q(t)] \Psi(q(0))\left[\left|q_{i}(T)\right|\right]^{\frac{1}{2}} \cos \left[\frac{1}{\hbar} \boldsymbol{S}_{1}(\dot{q}, q, \lambda, T)\right]=\int d y \breve{\mathcal{R}}_{1}(x, y, T, \lambda),(3.17) \\
\breve{\mathcal{R}}_{1}(x, y, T, \lambda)=\int_{\substack{q(T)=x \\
q(0)=y}} D^{+}[q(t)] \Psi(q(0))\left[\left|q_{i}(T)\right|\right]^{\frac{1}{2}} \cos \left[\frac{1}{\hbar} \boldsymbol{S}_{1}(\dot{q}, q, \lambda, T)\right](3.18)
\end{gathered}
$$

and

$$
\begin{gathered}
\breve{\mathcal{R}}_{2}(x, T, \lambda)=\int_{q(T)=x} D^{+}[q(t)] \Psi(q(0))\left[\left|q_{i}(T)\right|\right]^{\frac{1}{2}} \sin \left[\frac{1}{\hbar} \boldsymbol{S}_{1}(\dot{q}, q, \lambda, T)\right]= \\
=\int d y \int_{\substack{q(T)=x \\
q(0)=y}} D^{+}[q(t)] \Psi(q(0))\left[\left|q_{i}(T)\right|\right]^{\frac{1}{2}} \sin \left[\frac{1}{\hbar} \boldsymbol{S}_{1}(\dot{q}, q, \lambda, T)\right]=\int d y \breve{\mathcal{R}}_{2}(x, y, T, \lambda), \quad
\end{gathered}
$$


International Journal of Recent advances in Physics (IJRAP) Vol.3, No.4, November 2014

$\breve{\mathcal{R}}_{2}(x, y, T, \lambda)=\int_{\substack{q(T)=x \\ q(0)=y}} D^{+}[q(t)] \Psi(q(0))\left[\left|q_{i}(T)\right|\right]^{\frac{1}{2}} \sin \left[\frac{1}{\hbar} \boldsymbol{S}_{1}(\dot{q}, q, \lambda, T)\right] .(3.20)$

FromEq.(3.18) one obtain

$\breve{\mathcal{R}}_{1}(x, y, T, \lambda)=\frac{1}{2}\left[\breve{\mathcal{R}}_{1,1}(x, y, T, \lambda)+\breve{\mathcal{R}}_{1,2}(x, y, T, \lambda)\right],(3.21)$

where

$\breve{\mathcal{R}}_{1,1}(x, y, T, \lambda)=\int_{\substack{q(T)=x \\ q(0)=y}} D^{+}[q(t)] \Psi(q(0))\left[\left|q_{i}(T)\right|\right]^{\frac{1}{2}} \exp \left[\frac{i}{\hbar} \boldsymbol{S}_{1}(\dot{q}, q, \lambda, T)\right],(3.22)$

$\breve{\mathcal{R}}_{1,2}(x, y, T, \lambda)=\int_{\substack{q(T)=x \\ q(0)=y}} D^{+}[q(t)] \Psi(q(0))\left[\left|q_{i}(T)\right|\right]^{\frac{1}{2}} \exp \left[-\frac{i}{\hbar} \boldsymbol{S}_{1}(\dot{q}, q, \lambda, T)\right] .(3.23)$

Let us calculate now path integral $\breve{\mathcal{R}}_{1,1}(x, y, T, \lambda)$ and path integral $\breve{\mathcal{R}}_{1,2}(x, y, T, \lambda)$, using stationary phase approximation .From Eq.(A.23) follows directly that action $\boldsymbol{S}_{1}(\dot{q}, q, \lambda, T)$ coincide with master action $\mathrm{S}(t, \lambda, x, y)$ isgiven via formulae (3.6)-(3.8) and therefore from Eq.(3.22) and Eq.(3.23) one obtain

$$
\begin{gathered}
\breve{\mathcal{R}}_{1,1}(x, y, T, \lambda)=\int_{\substack{q(T)=x \\
q(0)=y}} D^{+}[q(t)] \Psi(q(0))\left[\left|q_{i}(T)\right|\right]^{\frac{1}{2}} \exp \left[\frac{i}{\hbar} \boldsymbol{S}_{1}(\dot{q}, q, \lambda, T)\right]= \\
=\breve{\mathcal{R}}_{1,1}(x, y, T, \lambda)=\left[\left|x_{i}\right|\right]^{\frac{1}{2}} \Psi(y) \exp \left[\frac{i}{\hbar} S(t, \lambda, x, y)\right](3.24)
\end{gathered}
$$

and

$$
\begin{gathered}
\breve{\mathcal{R}}_{1,2}(x, y, T, \lambda)=\int_{\substack{q(T)=x \\
q(0)=y}} D^{+}[q(t)] \Psi(q(0))\left[\left|q_{i}(T)\right|\right]^{\frac{1}{2}} \exp \left[\frac{i}{\hbar} \boldsymbol{S}_{1}(\dot{q}, q, \lambda, T)\right]= \\
=\breve{\mathcal{R}}_{1,2}(x, y, T, \lambda)=\left[\left|x_{i}\right|\right]^{\frac{1}{2}} \Psi(y) \exp \left[-\frac{i}{\hbar} S(t, \lambda, x, y)\right] \cdot(3.25)
\end{gathered}
$$

From Eq.(3.17) and Eq.(3.24) we obtain

$\breve{\mathcal{R}}_{1}(x, T, \lambda)=\int d y \breve{\mathcal{R}}_{1}(x, y, T, \lambda) \cdot(3.26)$

Substitution Eq.(3.25) into Eq.(3.26) gives

$\breve{\mathcal{R}}_{1,1}(x, T, \lambda)=\left[\left|x_{i}\right|\right]^{\frac{1}{2}} \int d y \Psi(y) \exp \left[\frac{i}{\hbar} S(t, \lambda, x, y)\right] .(3.26)$

Similarly one obtain

$\breve{\mathcal{R}}_{1,2}(x, T, \lambda)=\left[\left|x_{i}\right|\right]^{\frac{1}{2}} \int d y \Psi(y) \exp \left[-\frac{i}{\hbar} S(t, \lambda, x, y)\right] \cdot(3.27)$

Let us calculate now integral $\breve{\mathcal{R}}_{1,1}(x, T, \lambda)$ and integral $\breve{\mathcal{R}}_{1,2}(x, T, \lambda)$ using stationary phase approximation. Let $y_{c r}=y_{c r}(t, \lambda, x) \in \mathbb{R}^{d}$ be the stationary point of master action $S(t, \lambda, x, y)$ 
International Journal of Recent advances in Physics (IJRAP) Vol.3, No.4, November 2014 and therefore Eq.(3.9) is satisfied. Having applied stationary phase Approximation one obtain

$$
\begin{gathered}
\breve{\mathcal{R}}_{1,1}\left(x, y_{c r}(t, \lambda, x), T, \lambda\right)= \\
{\left[\left|\operatorname{det} S_{y_{c r} y_{c r}}\left(t, \lambda, x, y_{c r}(t, \lambda, x)\right)\right|\right]^{-\frac{1}{2}}\left[\left|x_{i}\right|\right]^{\frac{1}{2}} \Psi\left(y_{c r}(t, \lambda, x)\right) \exp \left[\frac{i}{\hbar} S\left(t, \lambda, x, y_{c r}(t, \lambda, x)\right)\right],(3.28)} \\
\breve{\mathcal{R}}_{1,2}\left(x, y_{c r}(t, \lambda, x), T, \lambda\right)= \\
{\left[\left|\operatorname{det} S_{y_{c r} y_{c r}}\left(t, \lambda, x, y_{c r}(t, \lambda, x)\right)\right|\right]^{-\frac{1}{2}}\left[\left|x_{i}\right|\right]^{\frac{1}{2}} \Psi\left(y_{c r}(t, \lambda, x)\right) \exp \left[-\frac{i}{\hbar} S\left(t, \lambda, x, y_{c r}(t, \lambda, x)\right)\right] .(3.29)}
\end{gathered}
$$

Substitution Eq.(3.28)-Eq.(3.29)into Eq.(3.21) gives

$$
\begin{gathered}
\breve{\mathcal{R}}_{1}\left(x, y_{c r}(t, \lambda, x), T, \lambda\right)=\frac{1}{2}\left[\breve{\mathcal{R}}_{1,1}\left(x, y_{c r}(t, \lambda, x), T, \lambda\right)+\breve{\mathcal{R}}_{1,2}\left(x, y_{c r}(t, \lambda, x), T, \lambda\right)\right]= \\
=\left[\left|\operatorname{det} S_{y_{c r} y_{c r}}\left(t, \lambda, x, y_{c r}(t, \lambda, x)\right)\right|\right]^{-\frac{1}{2}}\left[\left|x_{i}\right|\right]^{\frac{1}{2}} \Psi\left(y_{c r}(t, \lambda, x)\right) \times \\
\times\left\{\exp \left[\frac{i}{\hbar} S\left(t, \lambda, x, y_{c r}(t, \lambda, x)\right)\right]+\exp \left[-\frac{i}{\hbar} S\left(t, \lambda, x, y_{c r}(t, \lambda, x)\right)\right]\right\}= \\
=\left[\left|\operatorname{det} S_{y_{c r} y_{c r}}\left(t, \lambda, x, y_{c r}(t, \lambda, x)\right)\right|\right]^{-\frac{1}{2}}\left[\left|x_{i}\right|\right]^{\frac{1}{2}} \Psi\left(y_{c r}(t, \lambda, x)\right) \cos \left[\frac{1}{\hbar} S\left(t, \lambda, x, y_{c r}(t, \lambda, x)\right)\right] .(3.30)
\end{gathered}
$$

Substitution Eq.(3.30) into Eq.(3.16)gives

$$
\begin{gathered}
\mathcal{R}_{1}(T, \lambda)=\int d x \int d x\left[\breve{\mathcal{R}}_{1}(x, T, \lambda)\right]^{2}= \\
=\int d x\left[\left|\operatorname{det} S_{y_{c r} y_{c r}}\left(t, \lambda, x, y_{c r}(t, \lambda, x)\right)\right|\right]^{-1}\left|x_{i}\right| \Psi^{2}\left(y_{c r}(t, \lambda, x)\right) \cos ^{2}\left[\frac{1}{\hbar} S\left(t, \lambda, x, y_{c r}(t, \lambda, x)\right)\right] .
\end{gathered}
$$

Similarly one obtain

$$
\begin{gathered}
\mathcal{R}_{2}(T, \lambda)=\int d x \int d x\left[\breve{\mathcal{R}}_{2}(x, T, \lambda)\right]^{2}= \\
=\int d x\left[\left|\operatorname{det} S_{y_{c r} y_{c r}}\left(t, \lambda, x, y_{c r}(t, \lambda, x)\right)\right|\right]^{-1}\left|x_{i}\right| \Psi^{2}\left(y_{c r}(t, \lambda, x)\right) \sin ^{2}\left[\frac{1}{\hbar} S\left(t, \lambda, x, y_{c r}(t, \lambda, x)\right)\right] .
\end{gathered}
$$

Therefore

$\mathcal{R}(T, \lambda)=\mathcal{R}_{1}(T, \lambda)+\mathcal{R}_{2}(T, \lambda)=2 \int d x\left[\left|\operatorname{det} S_{y_{c r} y_{c r}}\left(t, \lambda, x, y_{c r}(t, \lambda, x)\right)\right|\right]^{-1}\left|x_{i}\right| \Psi^{2}\left(y_{c r}(t, \lambda, x)\right) .(3.33)$

Substitution Eq.(3.1) into Eq.(3.33) gives

$\mathcal{R}(T, \lambda)=2 \frac{\eta^{d / 2}}{(2 \pi)^{d / 2} \hbar^{d / 2}} \int d x\left[\left|\operatorname{det} S_{y_{c r} y_{c r}}\left(t, \lambda, x, y_{c r}(t, \lambda, x)\right)\right|\right]^{-1}\left|x_{i}\right| \exp \left[-\frac{\eta\left(y_{c r}(t, \lambda, x)-x_{0}\right)^{2}}{\hbar}\right](3.34)$

Let us calculate now integral (3.34)using Laplace's approximation.It is easy to see that corresponding stationary point $\hat{x}=\hat{x}\left(t, \lambda, x_{0}\right) \in \mathbb{R}^{d}$ is the solution of the linear system of the algebraic equations(3.10). Therefore finally we obtain 
International Journal of Recent advances in Physics (IJRAP) Vol.3, No.4, November 2014

$$
\mathcal{R}(T, \lambda)=2\left|\hat{x}_{i}\left(t, \lambda, x_{0}\right)\right|\left[\left|\operatorname{det} S_{y_{c r} y_{c r}}\left(t, \lambda, \hat{x}\left(t, \lambda, x_{0}\right), y_{c r}\left(t, \lambda, \hat{x}\left(t, \lambda, x_{0}\right)\right)\right)\right|\right]^{-1}+O\left(\hbar^{d}\right),
$$

$i=1, \ldots, d .(3.35)$

Substitution Eq.(3.35) into inequality (3.13) gives the inequality(3.11). The inequality (3.11) completed the proof.

\section{Quantum an harmonic oscillator with a cubic potential supplemented by additive sinusoidal driving.}

In this subsection we calculate exact quasi-classical asymptotic for quantum an harmonic oscillator with a cubic potential supplemented by additive sinusoidal driving. Using Theorem3.1 we obtain corresponding limiting quantum trajectories given via Eq.(1.3).

Let us consider quantum an harmonic oscillator with a cubic potential

$V(x)=\frac{m \omega^{2}}{2} x^{2}-a x^{3}+b x, x \in \mathbb{R}, a, b>0(4.1)$

supplemented by an additive sinusoidal driving. Thus

$$
V(x, t)=\frac{m \omega^{2}}{2} x^{2}-a x^{3}+b x-[A \sin (\Omega t)] x .
$$

The corresponding master Lagrangian given by(3.7), are

$L(\dot{u}, u, \tau)=\left(\frac{m}{2}\right) \dot{u}^{2}-m\left(\left(\frac{\omega^{2}}{2}\right)+\left(\frac{3 a \lambda}{m}\right)\right) u^{2}-\left(m \omega^{2} \lambda+3 a \lambda^{2}-b-A \sin (\Omega t)\right) u .(4.3)$

We assume now that: $\frac{\omega^{2}}{2}+\frac{3 a \lambda}{m} \geq 0$ and rewrite(4.3)of the form

$L(\dot{u}, u, \tau)=(m / 2) \dot{u}^{2}-\left(m \varpi^{2} \lambda / 2\right) u^{2}+g(\lambda, t) u$,

where $\varpi(\lambda)=\sqrt{2\left|\frac{\omega^{2}}{2}+\frac{3 a \lambda}{m}\right|}$ and $g(\lambda, t)=-\left[m \omega^{2} \lambda+3 a \lambda^{2}-b-A \sin (\Omega \cdot t)\right]$.

The corresponding master actionS $(t, \lambda, x, y)$ given by Eq.(3.6), are

$$
\begin{array}{r}
\mathrm{S}(t, \lambda, x, y)=\frac{m \varpi}{2 \sin \varpi t}\left[(\cos \varpi t)\left(y^{2}+x^{2}\right)-2 x y+\frac{2 x}{m \varpi} \int_{0}^{t} g(\lambda, \tau) \sin (\varpi \tau) d \tau+\right. \\
\left.+\frac{2 y}{m \varpi} \int_{0}^{t} g(\lambda, \tau) \sin (\varpi(t-\tau)) d \tau-\frac{2}{m^{2} \varpi^{2}} \int_{0}^{t} \int_{0}^{\tau} g(\lambda, \tau) g(\lambda, s) \sin \varpi(t-\tau) \sin (\varpi s) d s d \tau\right] .(4.5)
\end{array}
$$

The linear system of the algebraic equations(3.9) are

$\frac{\partial \mathrm{S}(t, \lambda, x, y)}{\partial y}=2 y \cos \varpi t-2 x+\frac{2}{m \varpi} \int_{0}^{t} g(\lambda, t) \sin ((\varpi(t-\tau)) d \tau=0$.

Therefore

$y_{c r}(t, \lambda, x)=\frac{x}{\cos \varpi t}-\frac{1}{m \varpi \cos \varpi t} \int_{0}^{t} g(\lambda, t) \sin ((\varpi(t-\tau)) d \tau$ (4.7) 
The linear system of the algebraic equations (3.10) are

$\frac{x}{\cos \varpi t}-\frac{1}{m \varpi \cos \varpi t} \int_{0}^{t} g(\lambda, t) \sin \left((\varpi(t-\tau)) d \tau+\lambda-x_{0}=0\right.$.

Therefore the solution of the linear system of the algebraic equations (3.10) are

$\hat{x}\left(t, \lambda, x_{0}\right)=\frac{1}{m \varpi} \int_{0}^{t} g(\lambda, t) \sin \left((\varpi(t-\tau)) d \tau+\left(\lambda(t)-x_{0}\right) \cos \varpi t\right.$.

Transcendental master equation (3.11) are

$\frac{1}{m \varpi} \int_{0}^{t} g(\lambda(t), t) \sin \left((\varpi(t-\tau)) d \tau+\left(\lambda(t)-x_{0}\right) \cos \varpi t=0\right.$

Finally from Eq.(4.10) one obtain

$d(\lambda(t))\left(\frac{\cos (\varpi t)}{\varpi}-\frac{1}{\varpi}\right)+\frac{A(\varpi \sin (\Omega t)-\Omega \sin (\varpi t))}{\varpi^{2}-\Omega^{2}}-\left(\lambda(t)-x_{0}\right) m \varpi \cos (\varpi t)=0,(4.11)$

whered $(\lambda)=m \omega^{2} \lambda+3 a \lambda^{2}-b$.

Numerical Examples.

Example1. $x_{0}=0, m=1, \Omega=0, \omega=9, a=3, b=10, A=0$.
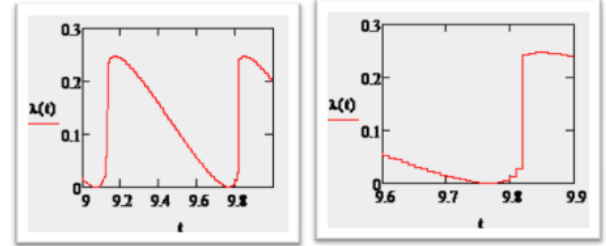

Pic.1. Limiting quantum trajectory $\lambda(t) P i c .2$. Limiting quantum trajectory $\lambda(t)$ with a jump

\section{Comparison exact quasi-classical asymptotic withstationary-point approximation.}

We set now $d=1$.Let us consider now path integral (2.14) with $S_{\varepsilon}(\dot{q}(t), q(t) ; t)$ given via formula

$\boldsymbol{S}_{\varepsilon}(\dot{q}(t), q(t), t)=\frac{1}{4} \int_{0}^{t}\left[\dot{q}(\tau)-F_{\varepsilon}(q(\tau), \tau)\right]^{2} d \tau$.

Note that for corresponding propagator $K_{\varepsilon}(x, t \mid y, 0)$ the time discretized path-integral representation

$K_{\varepsilon, N}(x, t \mid y, 0)$ are :

$K_{\varepsilon, N}(x, t \mid y, 0)=\int \frac{d x_{1} d x_{2} \ldots d x_{N-1}}{(4 \pi \hbar \Delta t)^{N / 2}} \exp \left[\frac{i}{\hbar} \boldsymbol{S}_{\varepsilon, N}\left(x_{0}, \ldots, x_{N}\right)\right],(5.2)$

where $S_{\varepsilon, N}\left(x_{0}, \ldots, x_{N}\right)$ are : 
International Journal of Recent advances in Physics (IJRAP) Vol.3, No.4, November 2014

$\boldsymbol{S}_{\varepsilon, N}\left(x_{0}, \ldots, x_{N}\right)=\frac{\Delta t}{4} \sum_{n=0}^{N-1}\left[\frac{x_{n+1}-x_{n}}{\Delta t}-F_{\varepsilon}\left(x_{n}, t_{n}\right)\right]^{2}$

Here the initial- $x_{0}$ and end-points $x_{N}$ are fixed by the prescribed $x_{0}$ and by the additional constraint $x_{N}=y$.

Let us calculate now integral (5.2) using stationary-point approximation. Denoting an critical points of the discrete-time action(5.3)by $\boldsymbol{x}_{\varepsilon, k}=\left(x_{1, \varepsilon, k}, \ldots, x_{N-1, \varepsilon, k}\right)$ it follows that $\boldsymbol{x}_{\varepsilon, k}$ satisfies the critical point conditions are

$\frac{\partial S_{\varepsilon, N}\left(x_{0, \varepsilon, k}, \ldots, x_{N-1, \varepsilon, k}, x_{N, \varepsilon, k}\right)}{\partial x_{n, \varepsilon, k}}=0(5.4)$

for $n=1, \ldots, N-1$, supplemented by the prescribed boundary conditions for $n=0, n=N: x_{0, \varepsilon, k}=$ $x_{0}$

$$
x_{N, \varepsilon, k}=x
$$

From Eq.(5.2) in the limit $\hbar \rightarrow$ 0using form all y Stationary-point approximation one obtain

$$
K_{\varepsilon, N}\left(x, t \mid x_{0}, 0\right) \cong Z_{\varepsilon, N-1}\left(\boldsymbol{x}_{\varepsilon, k}, x_{0, \varepsilon, k}, x_{N, \varepsilon, k}\right) \exp \left[\frac{i}{\hbar} \boldsymbol{S}_{\varepsilon, N}\left(\boldsymbol{x}_{\varepsilon, k}, x_{0, \varepsilon, k}, x_{N, \varepsilon, k}\right)\right]+O(\hbar) .(5.5)
$$

Here the pre-factor $\boldsymbol{Z}_{\varepsilon, N}\left(\boldsymbol{x}_{\varepsilon, k}\right)$ is given via N-dimensional Gaussian integral of the canonical form as

$$
\boldsymbol{Z}_{\varepsilon, N-1}\left(\boldsymbol{x}_{\varepsilon, k}, x_{0, \varepsilon, k}, x_{N, \varepsilon, k}\right)=\int \frac{d y_{1} d y_{2} \ldots d y_{N-1}}{(4 \pi \hbar \Delta t)^{N / 2}} \exp \left[\frac{i}{2 \hbar} \sum_{n, m=1}^{N-1} y_{n} \frac{\partial^{2} \boldsymbol{s}_{\varepsilon, N}\left(\boldsymbol{x}_{\varepsilon, k}, x_{0, \varepsilon, k}, x_{N, \varepsilon, k}\right)}{\partial x_{n, \varepsilon, k} \partial x_{m, \varepsilon, k}} y_{m}\right]
$$

The Gaussian integral in (5.6) is given via canonical formula

$\boldsymbol{Z}_{\varepsilon, N-1}\left(\boldsymbol{x}_{\varepsilon, k}, x_{0, \varepsilon, k}, x_{N, \varepsilon, k}\right)=\left[4 \pi \hbar \Delta t \operatorname{det}\left(2 \Delta t \frac{\partial^{2} s_{\varepsilon, N}\left(\boldsymbol{x}_{\varepsilon, k}, x_{0, \varepsilon, k}, x_{N, \varepsilon, k}\right)}{\partial x_{n, \varepsilon, k} \partial x_{m, \varepsilon, k}}\right)\right]^{-1 / 2}, n, m=1, \ldots, N-$

Heredet $\left(A_{n, m}\right)$ denotethe determinant of an $N-1 \times N-1$ matrix with elements $A_{n, m}$. Let us consider now Cauchy problem (2.8) with initial data $\Psi_{0}(x)$ is given via formula

$$
\Psi_{0}(x)=\frac{\eta^{1 / 4}}{(2 \pi)^{1 / 4} \hbar^{1 / 4}} \exp \left[-\frac{\eta\left(x-z_{0}\right)^{2}}{2 \hbar}\right]
$$

Note that for corresponding Colombeau solution $\Psi_{\varepsilon}(t, x)$ given viapath-integral(2.14)the time discretized path-integral representation $\Psi_{\varepsilon, N}(t, x)$ are

$$
\begin{gathered}
\Psi_{\varepsilon, N}(t, x)=\int \Psi_{0}\left(x_{0}\right) K_{\varepsilon, N}\left(x, t \mid x_{0}, 0\right) d x_{0}= \\
=\sum_{k} \int d x_{0} \boldsymbol{Z}_{\varepsilon, N-1}\left(\boldsymbol{x}_{\varepsilon, k}, x_{0}, x_{N, \varepsilon, k}\right) \Psi_{0}\left(x_{0}\right) \exp \left[\frac{i}{\hbar} \boldsymbol{S}_{\varepsilon, N}\left(\boldsymbol{x}_{\varepsilon, k}, x_{0}, x_{N, \varepsilon, k}\right)\right][1+O(\hbar)] .
\end{gathered}
$$

Let us calculate now integrals in RHS of Eq.(5.8) using stationary-point approximation. Corresponding critical point conditions are

$\frac{\partial S_{\varepsilon, N}\left(x_{0, \varepsilon, k}, \ldots, x_{N-1, \varepsilon, k}, x_{N, \varepsilon, k}\right)}{\partial x_{0, \varepsilon, k}}=0$. 
From (5.8) we obtain

$\Psi_{\varepsilon, N}(t, x)=\sum_{k} \boldsymbol{Z}_{\varepsilon, N}\left(\boldsymbol{x}_{\varepsilon, k}, x_{0, \varepsilon, k}, x\right) \Psi_{0}\left(x_{0, \varepsilon, k}\right) \exp \left[\frac{i}{\hbar} \boldsymbol{S}_{\varepsilon, N}\left(\boldsymbol{x}_{\varepsilon, k}, x_{0, \varepsilon, k}, x\right)\right][1+O(\hbar)]$.

$\boldsymbol{Z}_{\varepsilon, N}\left(\boldsymbol{x}_{\varepsilon, k}, x_{0, \varepsilon, k}, x\right)=\left[\Delta t \operatorname{det}\left(2 \Delta t \frac{\partial^{2} \boldsymbol{s}_{\varepsilon, N}\left(x_{\varepsilon, k}, x_{0, \varepsilon, k}, x\right)}{\partial x_{n, \varepsilon, k} \partial x_{m, \varepsilon, k}}\right)\right]^{-1 / 2}, n, m=0,1, \ldots, N-1$

Let as denote $\boldsymbol{x}_{\varepsilon, 0}=\left(x_{0, \varepsilon, 0}, x_{1, \varepsilon, 0}, \ldots, x_{N-1, \varepsilon, 0}\right)=\left(x_{0, \varepsilon, 0}(x), x_{1, \varepsilon, 0}(x), \ldots, x_{N-1, \varepsilon, 0}(x)\right)$ the critical point for which the critical point conditions(5.4) are

$\frac{x_{n+1, \varepsilon, 0}-x_{n, \varepsilon, 0}}{\Delta t}-F_{\varepsilon}\left(x_{n, \varepsilon, 0}, t_{n}\right)=0, n=0,1, \ldots, N-1 .(5.12)$

Therefore the time discretized path-integral representation of the Colom beau quantum averages given by Eq. (1.1) are

$$
\begin{aligned}
&\left(\left\langle 1, t, z_{0} ; \hbar, \varepsilon\right\rangle\right)_{\varepsilon}=\left(\int x\left|\Psi_{\varepsilon, N}(x, t ; \hbar)\right|^{2} d x\right)_{\varepsilon} \\
&=\frac{\eta^{1 / 2}}{(2 \pi)^{1 / 2} \hbar^{1 / 2}}\left(\int d x x \mathbf{Z}_{\varepsilon, N}^{2}\left(\boldsymbol{x}_{\varepsilon, k}, x_{0, \varepsilon, 0}, x\right) \exp \left[-\frac{\eta\left(x_{0, \varepsilon, 0}(x)-z_{0}\right)^{2}}{\hbar}\right]\right)_{\varepsilon}\left[1+O\left(\hbar^{2}\right)\right]+ \\
&+\frac{\eta^{1 / 2}}{(2 \pi)^{1 / 2} \hbar^{1 / 2}}\left(\sum_{k \geq 1} \int d x x \mathbf{Z}_{\varepsilon, N}^{2}\left(\boldsymbol{x}_{\varepsilon, k}, x_{0, \varepsilon, 0}, x\right) \exp \left[-\frac{\eta\left(x_{0, \varepsilon, 0}(\mathrm{x})-z_{0}\right)^{2}}{\hbar}\right]\right)_{\varepsilon}\left[1+O\left(\hbar^{2}\right)\right]+O\left(\exp \left(-c \hbar^{-1}\right)\right),(5.13)
\end{aligned}
$$

where $c>0, \varepsilon \in(0,1], x \in \mathbb{R}$.Let us calculate now integrals in RHS of Eq.(5.13) using stationary-point approximation. Corresponding critical point conditions are

$x_{0, \varepsilon, 0}\left(x_{N, \varepsilon, 0}\right)-z_{0}=0,(5.14)$.

$x_{0, \varepsilon, k}\left(x_{N, \varepsilon, k}\right)-z_{0}=0, k \geq 1$.(5.15).

Here $x_{N, \varepsilon, 0}$ can be calculated using linear recursion(5.12) with initial data $x_{0, \varepsilon, 0}=z_{0}$.

From Eq.(5.13)-Eq.(5.14) one obtain

$$
\begin{aligned}
& \left(\left\langle 1, t, z_{0} ; \hbar, \varepsilon\right\rangle\right)_{\varepsilon} \cong\left(x_{N, \varepsilon, 0} \boldsymbol{Z}_{\varepsilon, N}^{2}\left(\boldsymbol{x}_{\varepsilon, k}, x_{0, \varepsilon, 0}, x_{N, \varepsilon, 0}\right)\right)_{\varepsilon}(5.16) \\
& \operatorname{and} \boldsymbol{Z}_{\varepsilon, N}\left(\boldsymbol{x}_{\varepsilon, k}, x_{0, \varepsilon, k}, x_{N, \varepsilon, 0}\right)=\left[\Delta t \operatorname{det}\left(2 \Delta t \frac{\partial^{2} \boldsymbol{s}_{\varepsilon, N}\left(\boldsymbol{x}_{\varepsilon, k}, x_{0, \varepsilon, k}, x_{N, \varepsilon, 0}\right)}{\partial x_{n, \varepsilon, k} \partial x_{m, \varepsilon, k}}\right)\right]^{-1 / 2}, n, m=0,1, \ldots, N .
\end{aligned}
$$

As demonstrated in [24] the determinant appearing in(5.11) can be calculated using second Order linear recursion:

$$
\begin{gathered}
\frac{Q_{n+1, \varepsilon, k}-2 Q_{n, \varepsilon, k}-Q_{n-1, \varepsilon, k}}{(\Delta t)^{2}}=2 \frac{Q_{n, \varepsilon, k} F_{x}^{\prime}\left(x_{n, \varepsilon, k}, t_{n}\right)-Q_{n-1, \varepsilon, k} F_{x}^{\prime}\left(x_{n-1, \varepsilon, k}, t_{n-1}\right)}{\Delta t}- \\
-Q_{n, \varepsilon, k}\left[\frac{x_{n+1, \varepsilon, k}-x_{n, \varepsilon, k}}{\Delta t}-F_{\varepsilon}\left(x_{n, \varepsilon, k}, t_{n}\right)\right] F_{\varepsilon, x^{2}}^{\prime \prime}\left(x_{n, \varepsilon, k}, t_{n}\right)+ \\
+Q_{n, \varepsilon, k}\left[F_{\varepsilon, x}^{\prime}\left(x_{n, \varepsilon, k}, t_{n}\right)\right]^{2}-Q_{n-1, \varepsilon, k}\left[F_{\varepsilon, x}^{\prime}\left(x_{n-1, \varepsilon, k}, t_{n-1}\right)\right]^{2}, \\
F_{\varepsilon, x}^{\prime}(x, t)=\frac{\partial F_{\varepsilon}(x, t)}{\partial x}, F_{\varepsilon, x}^{\prime \prime}(x, t)=\frac{\partial^{2} F_{\varepsilon}(x, t)}{\partial x^{2}} \cdot(5.18)
\end{gathered}
$$


International Journal of Recent advances in Physics (IJRAP) Vol.3, No.4, November 2014

with initial data $Q_{1, \varepsilon, k}=\Delta t, Q_{2, \varepsilon, k}=Q_{1, \varepsilon, k}+\Delta t+O\left((\Delta t)^{2}\right)(5.19)$

from which the pre-factor $\boldsymbol{Z}_{\varepsilon, N}\left(\boldsymbol{x}_{\varepsilon, k}, x_{0, \varepsilon, k}, x_{N, \varepsilon, 0}\right)$ in (5.16)follows as

$\boldsymbol{Z}_{\varepsilon, N}\left(\boldsymbol{x}_{\varepsilon, k}, x_{0, \varepsilon, k}, x_{N, \varepsilon, 0}\right)=\sqrt{Q_{N, \varepsilon, k}} \cdot(5.20)$

In the limit $\Delta t \rightarrow 0$ from critical point conditions (5.12) and (5.14) one obtain

$\dot{x}(t)-F_{\varepsilon}(x(t), t)=0, x(0)=z_{0} \cdot(5.21)$

In the limit $\Delta t \rightarrow 0$ from a second order linear recursion(5.18)one obtain

the second order linear differential equation

$\ddot{Q}_{\varepsilon, 0}(t)=2 \frac{d}{d t}\left[Q_{\varepsilon, 0}(t) F_{\varepsilon, x}^{\prime}(x(t), t)\right](5.22)$

with initial data

$Q_{\varepsilon, 0}(0)=0, \dot{Q}_{\varepsilon, 0}(0)=1 .(5.23)$

By integration Eq.(5.22) one obtain the first order linear differential equation

$\dot{Q}_{\varepsilon, 0}(t)=2 Q_{\varepsilon, 0}(t) F_{\varepsilon, x}^{\prime}(x(t), t)+1, Q_{\varepsilon, 0}(0)=0 .(5.24)$

In the limit $\Delta t \rightarrow 0$ from Eq.(5.16), Eq.(5.20)-Eq.(5.21) and Eq.(5.24)one obtain

$E(t)=\left(\left\langle 1, t, z_{0} ; \hbar, \varepsilon\right\rangle\right)_{\varepsilon} \cong x(t) Q_{\varepsilon, 0}^{-2}(t) \cdot(5.25)$

We set now in Eq.(5.1)

$F_{\varepsilon}(q, \tau)=-a q^{3}+b x+A \sin (\Omega \tau) \cdot(5.26)$

Corresponding differential master equation are

$\dot{q}=-\left(3 a \lambda^{2}-b\right) q-\left(a \lambda^{3}-b \lambda\right)+A \sin (\Omega \tau), q(0)=z_{0}-\lambda .(5.27)$

From Eq.(5.27) one obtain that corresponding transcendental master equation are

$\left[z_{0}-\lambda(t)\right]-\frac{\Delta[\lambda(t)]}{\Theta[\lambda(t)]}\{\exp (t \Delta[\lambda(t)])-1\}+A \frac{\exp (t \Delta[\lambda(t)])}{\Omega^{2}+\Theta^{2}[\lambda(t)]}\{\Theta[\lambda(t)] \sin (\Omega \mathrm{t})-\Omega \cos (\Omega \mathrm{t})\}+$
$A \frac{\Omega}{\Omega^{2}+\Theta^{2}[\lambda(t)]}=0 .(5.28)$

Numerical Examples.

Comparison of the: (1)classical dynamics calculated by usingEq.(5.1) (red curve), (2)limiting quantum trajectory $\lambda(t)$ calculated byusingmaster equation Eq.(5.28) (blue curve) and (3) limiting quantum trajectorycalculated by usingstationary-point approximation given by Eq.(5.25)(green curve). 
International Journal of Recent advances in Physics (IJRAP) Vol.3, No.4, November 2014
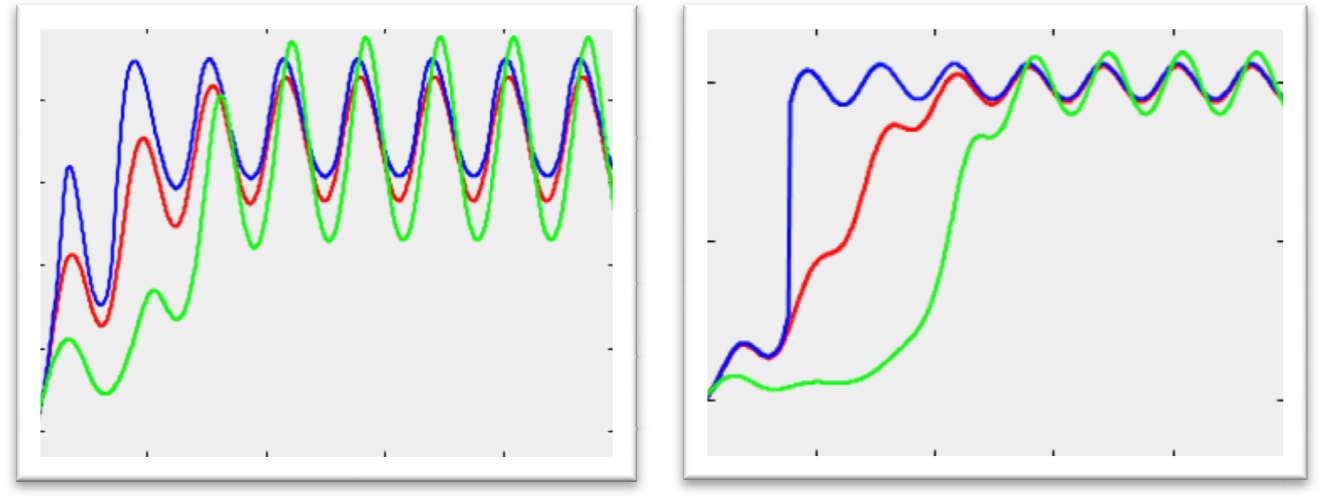

02468100246810

Pic.3.Limiting quantum trajectory $\lambda(t)$ Pic.4.Limiting quantum trajectory $\lambda(t)$ without jumps. $a=$ $0.3, b=1, A=2$.witha jump. $a=1, b=1, A=0.3$.

\section{Conclusions}

We pointed out that there exist limiting quantum trajectories given via Eq.(1.3) with a jumps. Such jumps does not depend on any single measurement of particle position $q(t)$ at instant $t$ and obtained without any reference to a phenomenological master-equation of Lind blad form. An axiom of quantum mechanics is that we cannot predict the result of any single measurement of an observableof a quantum mechanical system in a super position of eigen states. However we can predict the resultof any single measurement of particle position $q(t)$ at instant $t$ with a probability $P(t, q, \delta q) \cong 1$ if valid the condition: $\lim _{\varepsilon \rightarrow 0} \lim _{\|\delta q(t)\| \rightarrow 0} \lim _{\hbar \rightarrow 0} P_{t}\left(q(t), \delta q(t), x_{0} ; \hbar, \varepsilon\right)=1$, where $P_{t}$ given via Eq.(1.5).

\section{Acknowledgments}

A reviewer provided important clarification.

\section{Appendix}

Let us consider now regularized Feynman- Colombeau propagator $\left(K_{\varepsilon}(x, T \mid y, 0)\right)_{\varepsilon}$ given by Feynman path integral:

$\widetilde{K}_{\varepsilon}(x, T \mid y, 0 ; \sigma, l)=\int_{\substack{q(T)=x \\ q(0)=y}} D^{+}[q(t)] \exp \left[-\frac{1}{\hbar} \boldsymbol{S}_{1}(q, T ; \sigma, l)\right] \exp \left[-\frac{1}{\hbar} \boldsymbol{S}_{2}(q(T), \lambda)\right] \exp \left[\frac{i}{\hbar} \boldsymbol{S}_{\varepsilon}(\dot{q}, q, T)\right],(\mathrm{A} .1)$

where $\in \in(0,1]$,

$\boldsymbol{S}_{1}(q, T ; \sigma, l)=\int_{0}^{T} d t\left[\left\{[q(t)-\lambda]^{2} ; \sigma, l\right\}\right],(\mathrm{A} .2)$

$\boldsymbol{S}_{2}(q(T), \lambda)=[q(T)-\lambda]^{2}, \lambda=\left(\lambda_{1}, \ldots, \lambda_{d}\right) \in \mathbb{R}^{d},(\mathrm{~A} .3)$

$\boldsymbol{S}_{\varepsilon}(\dot{q}, q, T)=\int_{0}^{T} L(\dot{q}(t), q(t), t) d t, L(\dot{q}(t), q(t), t)=\frac{m}{2} \dot{q}^{2}(t)-V_{\varepsilon}(q(t), t), V_{\varepsilon=0}(x, t)=V(x, t),($ A. 4$)$

$V(x, t)=g_{1}(t) x+g_{2}(t) x^{2}+g_{3}(t) x^{3}+\cdots+g_{\alpha}(t) x^{\alpha},(\mathrm{A} .5)$

$$
\alpha=\left(i_{1}, \ldots, i_{d}\right), x^{\alpha}=x_{1}^{i_{1}} \times \ldots \times x_{d}^{i_{d}},\|\alpha\|=\sum_{r=1}^{d} i_{r}
$$


$V_{\varepsilon}(q(t), t)=V\left(q_{\varepsilon}(t), t\right), q_{\varepsilon}(t)=\left(q_{1, \varepsilon}(t), \ldots, q_{d, \varepsilon}(t)\right),($ A. 6$)$

$q_{i, \varepsilon}(t)=\frac{q_{i}(t)}{1+\varepsilon^{2 k}|q(t)|^{2 k}}, \varepsilon \in(0,1], k \geq 1$.(A.7)

Here: $(1) \sigma \in(0,1], \hbar \ll \sigma$ and (2) for each path $q(t)$ such

that $q(t)=\sum_{n=1}^{\infty} a_{n} \sin \left(\frac{n \pi t}{T}\right)+u(t, T, y, x), u(0, T, y, x)=y, u(T, T, y, x)=x$, where $u(t, T, y, x)$ is a given function, operator $\{p(t) ; \sigma, l\}$ are

$\{q(t) ; \sigma, l\}=\sum_{n=1}^{l} \sigma a_{n} \sin \left(\frac{n \pi t}{T}\right)+\sum_{n=l+1}^{\infty} a_{n} \sin \left(\frac{n \pi t}{T}\right) .(\mathrm{A} .8)$

(3) $D^{+}[q(t)]$ is a positive Feynman "measure".

Therefore regularized Colombeau solution of the Schrödinger equation corresponding to regularized propagator(A.1) are

$$
\begin{gathered}
\left(\Psi_{\varepsilon}(T, x ; \sigma, l, \lambda)\right)_{\varepsilon}=\left(\int_{-\infty}^{\infty} d y \Psi(y) \widetilde{K}_{\varepsilon}(x, T \mid y, 0 ; \sigma, l)\right)_{\varepsilon}= \\
\left(\int_{q(T)=x} D^{+}[q(t)] \Psi(q(0)) \exp \left[-\frac{1}{\hbar} \boldsymbol{S}(q, T ; \sigma, l, \lambda)\right] \exp \left[\frac{i}{\hbar} \boldsymbol{S}_{\varepsilon}(\dot{q}, q, T)\right]\right)_{\varepsilon}= \\
=\left(\int d y \int_{\substack{q(T)=x \\
q(0)=y}} D^{+}[q(t)] \Psi(q(0)) \exp \left[-\frac{1}{\hbar} \boldsymbol{S}(q, T ; \sigma, l, \lambda)\right] \exp \left[\frac{i}{\hbar} \boldsymbol{S}_{\varepsilon}(\dot{q}, q, T)\right]\right)_{\varepsilon} .(\text { A.9 } 9)
\end{gathered}
$$

$\operatorname{Here} \boldsymbol{S}(q, T ; \sigma, l, \lambda)=\boldsymbol{S}_{1}(q, T ; \sigma, l)+\boldsymbol{S}_{2}(q(T), \lambda) .(\mathrm{A} .10)$

Let us consider now regularized quantum average

$\left(\left\langle\hat{x}_{i}, T ; \sigma, l, \lambda, \varepsilon\right\rangle\right)_{\varepsilon}=\left(\int_{-\infty}^{\infty} d x x_{i}\left|\Psi_{\varepsilon}(T, x ; \sigma, l, \lambda)\right|^{2}\right)_{\varepsilon} \cdot(\mathrm{A} .11)$

From (A.5) and (A.11) one obtain

$$
\begin{aligned}
& \left(\left|\hat{x}_{i}, T ; \sigma, l, \lambda, \varepsilon\right|\right)_{\varepsilon} \\
& \leq\left(\int d x\left\{\int_{q(T)=x} D^{+}[q(t)] \Psi(q(0))\left[\left|q_{i}(T)\right|\right]^{1 / 2} \exp \left[-\frac{1}{\hbar} \boldsymbol{S}(q, T ; \sigma, l, \lambda)\right] \cos \left[\frac{1}{\hbar} \boldsymbol{S}_{\varepsilon}(\dot{q}, q, T)\right]\right\}^{2}\right)_{\varepsilon} \\
+ & \left(\int d x\left\{\int_{q(T)=x} D^{+}[q(t)] \Psi(q(0))\left[\left|q_{i}(T)\right|\right]^{1 / 2} \exp \left[-\frac{1}{\hbar} \boldsymbol{S}(q, T ; \sigma, l, \lambda)\right] \sin \left[\frac{1}{\hbar} \boldsymbol{S}_{\varepsilon}(\dot{q}, q, T)\right]\right\}^{2}\right)_{\varepsilon}(\mathrm{A} .13)
\end{aligned}
$$

From Eq.(A.5)-(A.13)one obtain

$$
\begin{array}{r}
\left|\left\langle\hat{x}_{i}, T ; \sigma, l, \lambda, \varepsilon\right\rangle-\lambda_{i}\right|=\left.\left|\left\langle\hat{x}_{i}, T ; \sigma, l, \lambda, \varepsilon\right\rangle-\lambda_{i} \int_{-\infty}^{\infty} d x\right| \Psi_{\varepsilon}(T, x ; \sigma, l, \lambda)\right|^{2} \mid= \\
=\left.\left|\int_{-\infty}^{\infty} d x\left[x_{i}-\lambda_{i}\right]\right| \Psi_{\varepsilon}(T, x ; \sigma, l, \lambda)\right|^{2}\left|\leq \int_{-\infty}^{\infty} d x\right| x_{i}-\left.\lambda_{i}|| \Psi_{\varepsilon}(T, x ; \sigma, l, \lambda)\right|^{2}=
\end{array}
$$


International Journal of Recent advances in Physics (IJRAP) Vol.3, No.4, November 2014

$$
\begin{gathered}
\quad=\int d x\left\{\int_{q(T)=x} D^{+}[q(t)] \Psi(q(0))\left[\left|q_{i}(T)-\lambda_{i}\right|\right]^{1 / 2} \exp \left[-\frac{1}{\hbar} \boldsymbol{S}(q, T ; \sigma, l, \lambda)\right] \cos \left[\frac{1}{\hbar} \boldsymbol{S}_{\varepsilon}(\dot{q}, q, T)\right]\right\}^{2}+ \\
+\int d x\left\{\int_{q(T)=x} D^{+}[q(t)] \Psi(q(0))\left[\left|q_{i}(T)-\lambda_{i}\right|\right]^{1 / 2} \exp \left[-\frac{1}{\hbar} \boldsymbol{S}(q, T ; \sigma, l, \lambda)\right] \sin \left[\frac{1}{\hbar} \boldsymbol{S}_{\varepsilon}(\dot{q}, q, T)\right]\right\}^{2}(\mathrm{~A} .14)
\end{gathered}
$$

Using replacement $q_{i}(t)-\lambda_{i}:=q_{i}(t), i=1, \ldots, d$ into RHS of the Eq.(A.9)one obtain

$$
\begin{gathered}
\left|\left\langle\hat{x}_{i}, T ; \sigma, l, \lambda, \varepsilon\right\rangle-\lambda_{i}\right| \leq \\
\leq \int d x\left\{\int_{q(T)=x} D^{+}[q(t)] \Psi(q(0))\left[\left|q_{i}(T)\right|\right]^{1 / 2} \exp \left[-\frac{1}{\hbar} \boldsymbol{S}(q, T ; \sigma, l)\right] \cos \left[\frac{1}{\hbar} \boldsymbol{S}_{\varepsilon}(\dot{q}, q+\lambda, T)\right]\right\}^{2}+ \\
+\int d x\left\{\int_{q(T)=x} D^{+}[q(t)] \Psi(q(0))\left[\left|q_{i}(T)\right|\right]^{1 / 2} \exp \left[-\frac{1}{\hbar} \boldsymbol{S}(q, T ; \sigma, l)\right] \sin \left[\frac{1}{\hbar} \boldsymbol{S}_{\varepsilon}(\dot{q}, q+\lambda, T)\right]\right\}^{2}= \\
=\int d x\left[I_{1}^{2}(x, T ; \sigma, l, \lambda, \varepsilon)\right]+\int d x\left[I_{2}^{2}(x, T ; \sigma, l, \lambda, \varepsilon)\right] .(\mathrm{A} .15)
\end{gathered}
$$

Here

$$
\boldsymbol{S}(q, T ; \sigma, l)=\boldsymbol{S}_{1}(q, T ; \sigma, l)+\boldsymbol{S}_{2}(q(T)), \boldsymbol{S}_{1}(q, T ; \sigma, l)=\int_{0}^{T} d t\left[\left\{[q(t)]^{2} ; \sigma, l\right\}\right]
$$

$\boldsymbol{S}_{2}(q(T))=[q(T)]^{2}, \lambda \in \mathbb{R}^{d}(\mathrm{~A} .16)$

and

$$
\begin{gathered}
I_{1}(x, T ; \sigma, l, \lambda, \varepsilon)= \\
=\int_{q(T)=x} D^{+}[q(t)] \Psi(q(0))\left[\left|q_{i}(T)\right|\right]^{1 / 2} \exp \left[-\frac{1}{\hbar} \boldsymbol{S}(q, T ; \sigma, l)\right] \cos \left[\frac{1}{\hbar} \boldsymbol{S}_{\varepsilon}(\dot{q}, q+\lambda, T)\right](\mathrm{A} .17) \\
I_{2}(x, T ; \sigma, l, \lambda, \varepsilon)= \\
=\int_{q(T)=x} D^{+}[q(t)] \Psi(q(0))\left[\left|q_{i}(T)\right|\right]^{1 / 2} \exp \left[-\frac{1}{\hbar} \boldsymbol{S}(q, T ; \sigma, l)\right] \sin \left[\frac{1}{\hbar} \boldsymbol{S}_{\varepsilon}(\dot{q}, q+\lambda, T)\right] .(\mathrm{A} .18)
\end{gathered}
$$

Let us rewrite a function $V_{\varepsilon}(q(t)+\lambda, t)$ in the following equivalent form:

$V_{\varepsilon}(q(t)+\lambda, t)=V_{\varepsilon, 0}(q(t), t, \lambda)+V_{\varepsilon, 1}(q(t), t, \lambda),($ A.19)

$V_{\varepsilon, 0}(q(t), t, \lambda)=a_{\varepsilon, 1}(q(t), t, \lambda) q(t)+a_{\varepsilon, 2}(q(t), t, \lambda) q^{2}(t),(\mathrm{A} .20)$

$V_{\varepsilon, 1}(q(t), t, \lambda)=a_{\varepsilon, 3}(q(t), t, \lambda) q^{3}(t)+\cdots+a_{\varepsilon, \alpha}(q(t), t, \lambda) q^{\alpha}(t),(\mathrm{A} .21)$

where $a_{\varepsilon=0,1}(q(t), t, \lambda)=c_{1}(t, \lambda), a_{\varepsilon=0,2}(q(t), t, \lambda)=c_{2}(t, \lambda), \ldots, a_{\varepsilon=0, \alpha}(q(t), t, \lambda)=c_{\alpha}(t, \lambda)$.

Let us evaluate now path integral $I_{1}(T ; \sigma, l, \lambda)$ given via Eq.(A.17). Substitution Eq.(A.19)into RHS of the Eq.(A.17) gives

$$
I_{1}(x, T ; \sigma, l, \lambda, \varepsilon)=I_{1}^{(1)}(x, T ; \sigma, l, \lambda, \varepsilon)+I_{1}^{(2)}(x, T ; \sigma, l, \lambda, \varepsilon)=
$$


International Journal of Recent advances in Physics (IJRAP) Vol.3, No.4, November 2014

$\int_{q(T)=x} D^{+}[q(t)] \Psi(q(0))\left[\left|q_{i}(T)\right|\right]^{\frac{1}{2}} \exp \left[-\frac{1}{\hbar} \boldsymbol{S}(q, T ; \sigma, l)\right] \cos \left[\frac{1}{\hbar} \boldsymbol{S}_{\varepsilon, 1}(\dot{q}, q+\lambda, T)\right] \cos \left[\frac{1}{\hbar} \boldsymbol{S}_{\varepsilon, 2}(q+\lambda, T)\right]+$ $+\int_{q(T)=x} D^{+}[q(t)] \Psi(q(0))\left[\left|q_{i}(T)\right|\right]^{1 / 2} \exp \left[-\frac{1}{\hbar} \boldsymbol{S}(q, T ; \sigma, l)\right] \sin \left[\frac{1}{\hbar} \boldsymbol{S}_{\varepsilon, 1}(\dot{q}, q, \lambda, T)\right] \sin \left[-\frac{1}{\hbar} \boldsymbol{S}_{\varepsilon, 2}(q, \lambda, T)\right],($ A.22.a)

$$
\begin{aligned}
& I_{2}(x, T ; \sigma, l, \lambda, \varepsilon)=I_{2}^{(1)}(x, T ; \sigma, l, \lambda, \varepsilon)+I_{2}^{(2)}(x, T ; \sigma, l, \lambda, \varepsilon)= \\
& \int_{q(T)=x} D^{+}[q(t)] \Psi(q(0))\left[\left|q_{i}(T)\right|\right]^{\frac{1}{2}} \exp \left[-\frac{1}{\hbar} \boldsymbol{S}(q, T ; \sigma, l)\right] \cos \left[\frac{1}{\hbar} \boldsymbol{S}_{\varepsilon, 1}(\dot{q}, q+\lambda, T)\right] \sin \left[\frac{1}{\hbar} \boldsymbol{S}_{\varepsilon, 2}(q+\lambda, T)\right]+ \\
& +\int_{q(T)=x} D^{+}[q(t)] \Psi(q(0))\left[\left|q_{i}(T)\right|\right]^{1 / 2} \exp \left[-\frac{1}{\hbar} \boldsymbol{S}(q, T ; \sigma, l)\right] \sin \left[\frac{1}{\hbar} \boldsymbol{S}_{\varepsilon, 1}(\dot{q}, q, \lambda, T)\right] \cos \left[-\frac{1}{\hbar} \boldsymbol{S}_{\varepsilon, 2}(q, \lambda, T)\right] \\
& \text { (A.22.b) }
\end{aligned}
$$

where

$\boldsymbol{S}_{\varepsilon, 1}(\dot{q}, q, \lambda, T)=\int_{0}^{T} L_{\varepsilon}(\dot{q}(t), q(t), t, \lambda) d t, L_{\varepsilon}(\dot{q}(t), q(t), t, \lambda)=\frac{m}{2} \dot{q}^{2}(t)-V_{\varepsilon, 0}(q(t), t, \lambda),(\mathrm{A} .23)$

$\boldsymbol{S}_{\varepsilon, 2}(q, \lambda, T)=\int_{0}^{T} V_{\varepsilon, 1}(q(t), t, \lambda) d t,(\mathrm{~A} .24)$

$$
I_{1}^{(1)}(x, T ; \sigma, l, \lambda, \varepsilon)=
$$

$\int_{q(T)=x} D^{+}[q(t)] \Psi(q(0))\left[\left|q_{i}(T)\right|\right]^{\frac{1}{2}} \exp \left[-\frac{1}{\hbar} \boldsymbol{S}(q, T ; \sigma, l)\right] \cos \left[\frac{1}{\hbar} \boldsymbol{S}_{\varepsilon, 1}(\dot{q}, q, \lambda, T)\right] \cos \left[\frac{1}{\hbar} \boldsymbol{S}_{\varepsilon, 2}(q, \lambda, T)\right],(\mathrm{A} .25$.

a)

$$
I_{2}^{(1)}(x, T ; \sigma, l, \lambda, \varepsilon)=
$$

$\int_{q(T)=x} D^{+}[q(t)] \Psi(q(0))\left[\left|q_{i}(T)\right|\right]^{\frac{1}{2}} \exp \left[-\frac{1}{\hbar} \boldsymbol{S}(q, T ; \sigma, l)\right] \cos \left[\frac{1}{\hbar} \boldsymbol{S}_{\varepsilon, 1}(\dot{q}, q, \lambda, T)\right] \sin \left[\frac{1}{\hbar} \boldsymbol{S}_{\varepsilon, 2}(q, \lambda, T)\right]$ (A.25.b)

$$
I_{1}^{(2)}(x, T ; \sigma, l, \lambda, \varepsilon)=
$$

$\int_{q(T)=x} D^{+}[q(t)] \Psi(q(0))\left[\left|q_{i}(T)\right|\right]^{1 / 2} \exp \left[-\frac{1}{\hbar} \boldsymbol{S}(q, T ; \sigma, l)\right] \sin \left[\frac{1}{\hbar} \boldsymbol{S}_{\varepsilon, 1}(\dot{q}, q, \lambda, T)\right] \sin \left[-\frac{1}{\hbar} \boldsymbol{S}_{\varepsilon, 2}(q, \lambda, T)\right] .(\mathrm{A} .2$ 6.a)

$$
I_{2}^{(2)}(x, T ; \sigma, l, \lambda, \varepsilon)=
$$

$\int_{q(T)=x} D^{+}[q(t)] \Psi(q(0))\left[\left|q_{i}(T)\right|\right]^{1 / 2} \exp \left[-\frac{1}{\hbar} \boldsymbol{S}(q, T ; \sigma, l)\right] \sin \left[\frac{1}{\hbar} \boldsymbol{S}_{\varepsilon, 1}(\dot{q}, q, \lambda, T)\right] \cos \left[-\frac{1}{\hbar} \boldsymbol{S}_{\varepsilon, 2}(q, \lambda, T)\right]$. (A.26.b)

Let us evaluate nown-dimensional path integral $I_{1, n}^{(1)}(x, T ; \sigma, l, \lambda, \varepsilon)$ :

$$
I_{1, n}^{(1)}(x, T ; \sigma, l, \lambda, \varepsilon)=
$$


International Journal of Recent advances in Physics (IJRAP) Vol.3, No.4, November 2014

$$
\begin{gathered}
=\int_{q(T)=x} D_{n}^{+}[q(t)] \Psi(q(0))\left[\left|q_{i}(T)\right|\right]^{\frac{1}{2}} \exp \left[-\frac{1}{\hbar} \boldsymbol{S}(q, T ; \sigma, l)\right] \cos \left[\frac{1}{\hbar} \boldsymbol{S}_{\varepsilon, 1}(\dot{q}, q, \lambda, T)\right] \cos \left[\frac{1}{\hbar} \boldsymbol{S}_{\varepsilon, 2}(q, \lambda, T)\right]= \\
=\int_{q(T)=x} D_{n}^{+}[q(t)] \Psi(q(0))\left[\left|q_{i}(T)\right|\right]^{\frac{1}{2}} \exp \left[-\frac{1}{\hbar} \boldsymbol{S}(q, T ; \sigma, l)\right]\left\{\cos \left[\frac{1}{\hbar} S_{\varepsilon, 1}(\dot{q}, q, \lambda, T)\right]\right. \\
+1\} \cos \left[\frac{1}{\hbar} \boldsymbol{S}_{\varepsilon, 2}(q, \lambda, T)\right]-
\end{gathered}
$$$$
-\int_{q(T)=x} D_{n}^{+}[q(t)] \Psi(q(0))\left[\left|q_{i}(T)\right|\right]^{\frac{1}{2}} \exp \left[-\frac{1}{\hbar} \boldsymbol{S}(q, T ; \sigma, l)\right] \cos \left[\frac{1}{\hbar} \boldsymbol{S}_{\varepsilon, 2}(q, \lambda, T)\right] .(\mathrm{A} .27)
$$

From Eq.(A.27) one obtain the inequality

$$
\begin{aligned}
& \left|I_{1, n}^{(1)}(x, T ; \sigma, l, \lambda, \varepsilon)\right| \\
& \leq\left|\int D_{n}^{+}[q(t)] \Psi(q(0))\left[\left|q_{i}(T)\right|\right]^{\frac{1}{2}} \exp \left[-\frac{1}{\hbar} \boldsymbol{S}(q, T ; \sigma, l)\right]\left\{\cos \left[\frac{1}{\hbar} \boldsymbol{S}_{\varepsilon, 1}(\dot{q}, q, \lambda, T)\right]+1\right\}\right|- \\
& -\int_{q(T)=x} D_{n}^{+}[q(t)] \Psi(q(0))\left[\left|q_{i}(T)\right|\right]^{\frac{1}{2}} \exp \left[-\frac{1}{\hbar} \boldsymbol{S}(q, T ; \sigma, l)\right] \cos \left[\frac{1}{\hbar} \boldsymbol{S}_{\varepsilon, 2}(q, \lambda, T)\right]= \\
& =\left|\int_{q(T)=x} D_{n}^{+}[q(t)] \Psi(q(0))\left[\left|q_{i}(T)\right|\right]^{\frac{1}{2}} \exp \left[-\frac{1}{\hbar} \boldsymbol{S}(q, T ; \sigma, l)\right] \cos \left[\frac{1}{\hbar} \boldsymbol{S}_{\varepsilon, 1}(\dot{q}, q, \lambda, T)\right]\right|+ \\
& +\int_{q(T)=x} D_{n}^{+}[q(t)] \Psi(q(0))\left[\left|q_{i}(T)\right|\right]^{\frac{1}{2}} \exp \left[-\frac{1}{\hbar} \boldsymbol{S}(q, T ; \sigma, l)\right]- \\
& -\int_{q(T)=x} D_{n}^{+}[q(t)] \Psi(q(0))\left[\left|q_{i}(T)\right|\right]^{\frac{1}{2}} \exp \left[-\frac{1}{\hbar} \boldsymbol{S}(q, T ; \sigma, l)\right] \cos \left[\frac{1}{\hbar} \boldsymbol{S}_{\varepsilon, 2}(q, \lambda, T)\right] .(\mathrm{A} .28)
\end{aligned}
$$

From Inq.(A.28) one obtain the inequality

$$
\begin{aligned}
& \begin{array}{l}
\left|I_{1, n}^{(1)}(x, T ; \sigma, l, \lambda, \varepsilon)\right| \leq\left|\int_{q(T)=x} D_{n}^{+}[q(t)] \Psi(q(0))\left[\left|q_{i}(T)\right|\right]^{\frac{1}{2}} \exp \left[-\frac{1}{\hbar} \boldsymbol{S}(q, T ; \sigma, l)\right] \cos \left[\frac{1}{\hbar} \boldsymbol{S}_{\varepsilon, 1}(\dot{q}, q, \lambda, T)\right]\right|- \\
\quad-\sum_{i=1}^{\infty} \frac{(-1)^{i} \hbar^{-2 i}}{(2 i) !} \int_{q(T)=x} D_{n}^{+}[q(t)] \Psi(q(0))\left[\left|q_{i}(T)\right|\right]^{\frac{1}{2}}\left[\boldsymbol{S}_{\varepsilon, 2}(q, \lambda, T)\right]^{2 i} \exp \left[-\frac{1}{\hbar} \boldsymbol{S}(q, T ; \sigma, l)\right]= \\
=\left|\mathbf{J}_{1, n}^{(1)}(x, T ; \sigma, l, \lambda, \varepsilon, \hbar)\right|-\sum_{i=1}^{\infty} \frac{(-1)^{i} \hbar^{-2 i}}{(2 i) !} \mathcal{R}_{\varepsilon}^{(i)}(x, T ; \sigma, l, n),(\mathrm{A} .29)
\end{array} \\
& \text { where }
\end{aligned}
$$

$$
\mathbf{J}_{1, n}^{(1)}(x, T ; \sigma, l, \lambda, \varepsilon, \hbar)=
$$


International Journal of Recent advances in Physics (IJRAP) Vol.3, No.4, November 2014 $=\int_{q(T)=x} D_{n}^{+}[q(t)] \Psi(q(0))\left[\left|q_{i}(T)\right|\right]^{\frac{1}{2}} \exp \left[-\frac{1}{\hbar} \boldsymbol{S}(q, T ; \sigma, l)\right] \cos \left[\frac{1}{\hbar} \boldsymbol{S}_{\varepsilon, 1}(\dot{q}, q, \lambda, T)\right],(\mathrm{A} .30)$ $\mathcal{R}_{\varepsilon}^{(i)}(x, T ; \sigma, l, n)=\int_{q(T)=x} D_{n}^{+}[q(t)] \Psi(q(0))\left[\left|q_{i}(T)\right|\right]^{\frac{1}{2}}\left[\boldsymbol{S}_{\varepsilon, 2}(q, \lambda, T)\right]^{2 i} \exp \left[-\frac{1}{\hbar} \boldsymbol{S}(q, T ; \sigma, l)\right] .(\mathrm{A} .31)$ Using replacement $q_{i}(t):=\hbar^{\frac{1}{2}} q_{i}(t), t \in[0, T], i=1, \ldots, d$ into RHS of the Eq.(A.31) one obtain

$$
\begin{aligned}
& \mathcal{R}_{\varepsilon}^{(i)}(x, T ; \sigma, l, n) \\
& =\hbar^{1 / 4} \int_{q(T)=\frac{x}{\sqrt{\hbar}}} \breve{D}_{n}^{+}[q(t)] \Psi\left(\hbar^{\frac{1}{2}} q(0)\right)\left[\left|q_{i}(T)\right|\right]^{\frac{1}{2}}\left[\boldsymbol{S}_{\varepsilon, 2}\left(\hbar^{1 / 2} q, \lambda, T\right)\right]^{2 i} \exp \left[-\frac{1}{\hbar} \boldsymbol{S}\left(\hbar^{1 / 2} q, T ; \sigma, l\right)\right]= \\
& \hbar^{1 / 4} \hbar^{i / 2} \int d y \int_{q(T)=\frac{x}{\sqrt{\hbar}}} \breve{D}_{n}^{+}[q(t)] \breve{\Psi}(q(0))\left[\left|q_{i}(T)\right|\right]^{\frac{1}{2}}\left[\widehat{\boldsymbol{S}}_{\varepsilon, 2}(q, \lambda, T, \hbar)\right]^{2 i} \exp [-\boldsymbol{S}(q, T ; \sigma, l)]= \\
& =\hbar^{1 / 4} \hbar^{i / 2} \widehat{\mathcal{R}}_{\varepsilon}^{(i)}(x, T ; \sigma, l, n), \text { where }(\mathrm{A} .32) \\
& \breve{D}_{n}^{+}[q(t)]=D_{n}^{+}\left[\hbar^{\frac{1}{2}} q(t)\right], t \in[0, T], \widetilde{\Psi}(q(0))=\frac{\eta^{d / 4}}{(2 \pi)^{d / 4} \hbar^{d / 4}} \exp \left[\frac{\eta q^{2}(0)}{2}\right], \text { see Eq. (3.1) and } \\
& \widehat{\boldsymbol{S}}_{\varepsilon, 2}(q, \lambda, T, \hbar)=\int_{0}^{T} \widehat{V}_{\varepsilon, 1}(q(t), t, \lambda, \hbar) d t,(\mathrm{~A} .33) \\
& \widehat{V}_{\varepsilon, 1}(q(t), t, \lambda, \hbar)=a_{\varepsilon, 3}(q(t), t, \lambda) q^{3}(t)+\cdots+\hbar^{\frac{\alpha-3}{2}} a_{\varepsilon, \alpha}(q(t), t, \lambda) q^{\alpha}(t) .(\mathrm{A} .34) \\
& \hat{\mathcal{R}}_{\varepsilon}^{(i)}(x, T ; \sigma, l, n)=\int d y \int_{q(T)=\frac{x}{\sqrt{\hbar}}} \breve{D}_{n}^{+}[q(t)] \breve{\Psi}(q(0))\left[\left|q_{i}(T)\right|\right]^{\frac{1}{2}}\left[\widehat{\boldsymbol{S}}_{\varepsilon, 2}(q, \lambda, T, \hbar)\right]^{2 i} \exp [-\boldsymbol{S}(q, T ; \sigma, l)] .(\mathrm{A} . \\
& q(0)=\frac{y}{\sqrt{\hbar}}
\end{aligned}
$$

From (A.29)-(A.35) one obtain

$$
\left|I_{1, n}^{(1)}(x, T ; \sigma, l, \lambda, \varepsilon)\right| \leq\left|\mathbf{J}_{1, n}^{(1)}(x, T ; \sigma, l, \lambda, \varepsilon, \hbar)\right|-\hbar^{\frac{1}{4}} \sum_{i=1}^{\infty} \frac{(-1)^{i} \hbar^{i}}{(2 i) !} \widehat{\mathcal{R}}_{\varepsilon}^{(i)}(x, T ; \sigma, l, n)=
$$

$\leq\left|\mathbf{J}_{1, n}^{(1)}(x, T ; \sigma, l, \lambda, \varepsilon, \hbar)\right|-\hbar^{\frac{1}{4}} \Xi_{\varepsilon}(x, T ; \sigma, l, n)$, where(A.36)

$\Xi_{\varepsilon}(x, T ; \sigma, l, \hbar, n)=\sum_{i=1}^{\infty} \frac{(-1)^{i} \hbar^{i}}{(2 i) !} \hat{\mathcal{R}}_{\varepsilon}^{(i)}(x, T ; \sigma, l, n) .(\mathrm{A} .37)$

Proposition A.1.[21]-[23]Let $\left\{s_{n, m}\right\}_{n, m=1}^{n, m=\infty}$ be a double sequences: $\mathbb{N} \times \mathbb{N} \rightarrow \mathbb{C} . \operatorname{Letlim}_{n, m \rightarrow \infty} s_{n, m}=a$.

Then the iterated $\operatorname{limit} \lim _{n \rightarrow \infty}\left(\lim _{m \rightarrow \infty} s_{n, m}\right)$ exist and equal to $a$ if and only iflim ${ }_{m \rightarrow \infty} s_{n, m}$ exists for each $n \in \mathbb{N}$.

Proposition A.2.Let $I_{1}^{(1)}(x, T ; \sigma, l, \lambda, \varepsilon, \hbar)=I_{1}^{(1)}(x, T ; \sigma, l, \lambda, \varepsilon)$, where $I_{1}^{(1)}(x, T ; \sigma, l, \lambda, \varepsilon)$ is given via 
International Journal of Recent advances in Physics (IJRAP) Vol.3, No.4, November 2014 Eq.(A.25) and let $I_{1}^{(2)}(x, T ; \sigma, l, \lambda, \varepsilon, \hbar)=I_{1}^{(2)}(x, T ; \sigma, l, \lambda, \varepsilon)$, where $I_{1}^{(2)}(x, T ; \sigma, l, \lambda, \varepsilon)$ is given via Eq.(A.26). Then $I_{2}^{(2)}(x, T ; \sigma, l, \lambda, \varepsilon)=$

(1) $\underline{\lim }_{\boldsymbol{\varepsilon} \rightarrow 0} \underline{\lim }_{\sigma \rightarrow 0} \int d x\left[I_{1}^{(1)}(x, T ; \sigma, l, \lambda, \varepsilon)\right]^{2} \leq$

$$
\leq \lim _{\hbar \rightarrow 0} \int d x\left\{\int_{q(T)=x} D^{+}[q(t)] \Psi(q(0))\left[\left|q_{i}(T)\right|\right]^{\frac{1}{2}} \cos \left[\frac{1}{\hbar} \boldsymbol{S}_{1}(\dot{q}, q, \lambda, T)\right]\right\}^{2}
$$

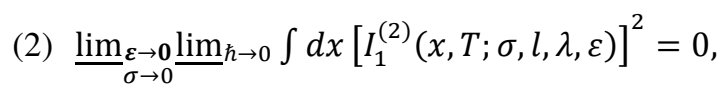

(3) $\underline{\lim }_{\substack{\varepsilon \rightarrow 0 \\ \sigma \rightarrow 0}} \lim _{\hbar \rightarrow 0} \int d x\left[I_{1}^{(1)}(x, T ; \sigma, l, \lambda, \varepsilon) I_{1}^{(2)}(x, T ; \sigma, l, \lambda, \varepsilon)\right]=0$,

(4) $\underline{\lim }_{\substack{\varepsilon \rightarrow 0 \\ \sigma \rightarrow 0}} \underline{\lim }_{\hbar \rightarrow 0} \int d x\left[I_{2}^{(2)}(x, T ; \sigma, l, \lambda, \varepsilon)\right]^{2} \leq$

$$
\leq \lim _{\hbar \rightarrow 0} \int d x\left\{\int_{q(T)=x} D^{+}[q(t)] \Psi(q(0))\left[\left|q_{i}(T)\right|\right]^{\frac{1}{2}} \sin \left[\frac{1}{\hbar} \boldsymbol{S}_{1}(\dot{q}, q, \lambda, T)\right]\right\}^{2},
$$

(5) $\underline{\lim }_{\substack{\varepsilon \rightarrow 0 \\ \sigma \rightarrow 0}} \underline{\lim }_{\hbar \rightarrow 0} \int d x\left[I_{2}^{(1)}(x, T ; \sigma, l, \lambda, \varepsilon)\right]^{2}=0$,

(6) $\underline{\lim }_{\sigma \rightarrow 0} \underline{\lim }_{\sigma \rightarrow 0} \int d x\left[I_{2}^{(2)}(x, T ; \sigma, l, \lambda, \varepsilon) I_{2}^{(1)}(x, T ; \sigma, l, \lambda, \varepsilon)\right]=0$.

Here

$$
\begin{gathered}
\boldsymbol{S}_{\mathbf{1}}(\dot{q}, q, \lambda, T)=\boldsymbol{S}_{\varepsilon=0,1}(\dot{q}, q, \lambda, T)=\int_{0}^{T} L_{\varepsilon=0}(\dot{q}(t), q(t), t, \lambda) d t \\
L_{\varepsilon=0}(\dot{q}(t), q(t), t, \lambda)=\frac{m}{2} \dot{q}^{2}(t)-V_{\varepsilon=0,0}(q(t), t, \lambda) .
\end{gathered}
$$

$\operatorname{Proof}(\mathbf{I})$ Let us to choose an sequence $\left\{\hbar_{m}\right\}_{m=1}^{\infty}$ such that

(i) $\quad \lim _{m \rightarrow \infty} \hbar_{m}=0$ and

(ii) $\quad \lim _{\mathrm{m}, \mathrm{n} \rightarrow \infty} \int d x\left\{\mathbf{\Xi}_{\varepsilon}^{(m)}\left(x, T ; \sigma, l, \hbar_{m}, n\right)\right\}^{2}=\lim _{m, n \rightarrow \infty} \int d x\left\{\sum_{i=1}^{m} \frac{(-1)^{i} \hbar_{m}^{i}}{(2 i) !} \hat{\mathcal{R}}_{\varepsilon}^{(i)}(x, T ; \sigma, l, n)\right\}^{2}=0$.

We note that from (ii) follows that: perturbative expansion

$$
\int d x\left\{\boldsymbol{\Xi}_{\varepsilon}\left(x, T ; \sigma, l, \hbar_{m}, n\right)\right\}^{2}=\hbar_{m}^{1 / 4} \int d x\left\{\sum_{i=1}^{\infty} \frac{(-1)^{i} \hbar_{m}^{i}}{(2 i) !} \hat{\mathcal{R}}_{\varepsilon}^{(i)}(x, T ; \sigma, l, n)\right\}^{2}
$$


International Journal of Recent advances in Physics (IJRAP) Vol.3, No.4, November 2014 vanishes in the limit $m, n \rightarrow \infty$.From (A.36) and Schwarz'sinequality using Proposition A.1,one obtain

$$
\begin{aligned}
& \underline{\lim }_{m, n \rightarrow \infty} \int d x\left[I_{1, n}^{(1)}\left(x, T ; \sigma, l, \lambda, \varepsilon, \hbar_{m}\right)\right]^{2} \\
& \leq \underline{\lim }_{m, n \rightarrow \infty} \int d x\left\{\left|\mathbf{J}_{1, n}^{(1)}\left(x, T ; \sigma, l, \lambda, \varepsilon, \hbar_{m}\right)\right|-\hbar_{m}^{1 / 4} \Xi_{\varepsilon}(x, T ; \sigma, l, \hbar, n)\right\}^{2} \\
& \leq \varlimsup_{m, n \rightarrow \infty} \int d x\left\{\mathbf{J}_{1, n}^{(1)}\left(x, T ; \sigma, l, \lambda, \varepsilon, \hbar_{m}\right)\right\}^{2}+ \\
& +\underline{\lim }_{m, n \rightarrow \infty}\left\{2 \hbar_{m}^{1 / 4} \sqrt{\left[\int d x\left\{\mathbf{J}_{1, n}^{(1)}\left(x, T ; \sigma, l, \lambda, \varepsilon, \hbar_{m}\right)\right\}^{2} \int d x\left\{\boldsymbol{\Xi}_{\varepsilon}\left(x, T ; \sigma, l, \hbar_{m}, n\right)\right\}^{2}\right]}\right. \\
& \left.+\int d x\left\{\boldsymbol{\Xi}_{\varepsilon}\left(x, T ; \sigma, l, \hbar_{m}, n\right)\right\}^{2}\right\} \\
& =\lim _{m, n \rightarrow \infty} \int d x\left\{\mathbf{J}_{1, n}^{(1)}\left(x, T ; \sigma, l, \lambda, \varepsilon, \hbar_{m}\right)\right\}^{2}=\lim _{\hbar \rightarrow 0} \lim _{n \rightarrow \infty} \int d x\left\{\mathbf{J}_{1, n}^{(1)}(x, T ; \sigma, l, \lambda, \varepsilon, \hbar)\right\}^{2}= \\
& =\lim _{\hbar \rightarrow 0} \int d x\left\{\int_{q(T)=x} D^{+}[q(t)] \Psi(q(0))\left[\left|q_{i}(T)\right|\right]^{\frac{1}{2}} \cos \left[\frac{1}{\hbar} \boldsymbol{S}_{1}(\dot{q}, q, \lambda, T)\right]\right\}^{2} \text {.(A.38) }
\end{aligned}
$$

Let us to choose now an subsequence $\left\{\hbar_{m_{k}}\right\}_{m_{k}=1}^{\infty}$ such that the limit: $\lim _{k, n \rightarrow \infty} \int d x\left[I_{1, n}^{(1)}\left(x, T ; \sigma, l, \lambda, \varepsilon, \hbar_{m_{k}}\right)\right]^{2}$ exist and $\lim _{\mathrm{k}, \mathrm{n} \rightarrow \infty} \int d x\left[I_{1, n}^{(1)}\left(x, T ; \sigma, l, \lambda, \varepsilon, \hbar_{m_{k}}\right)\right]^{2}=$ $\underline{\lim }_{m, n \rightarrow \infty} \int d x\left[I_{1, n}^{(1)}\left(x, T ; \sigma, l, \lambda, \varepsilon, \hbar_{m}\right)\right]^{2}(\mathrm{~A} .39)$

From (A.39) andProposition A.1 one obtain

$\lim _{\mathrm{k}, \mathrm{n} \rightarrow \infty} \int d x\left[I_{1, n}^{(1)}\left(x, T ; \sigma, l, \lambda, \varepsilon, \hbar_{m_{k}}\right)\right]^{2}=\lim _{k \rightarrow \infty}\left\{\lim \mathrm{n} \rightarrow \infty \int d x\left[I_{1, n}^{(1)}\left(x, T ; \sigma, l, \lambda, \varepsilon, \hbar_{m_{k}}\right)\right]^{2}\right\} .(\mathrm{A}$

From (A.39),(A.40) and(A.38)one obtain

$$
\begin{gathered}
\underline{\lim }_{\substack{\varepsilon \rightarrow 0 \\
\sigma \rightarrow 0}} \underline{\lim }_{\hbar \rightarrow 0} \int d x\left[I_{1}^{(1)}(x, T ; \sigma, l, \lambda, \varepsilon)\right]^{2} \leq \\
\leq \underline{\lim }_{\substack{\varepsilon \rightarrow 0 \\
\sigma \rightarrow 0}} \lim _{\mathrm{k} \rightarrow \infty}\left\{\lim \mathrm{n} \rightarrow \infty \int d x\left[I_{1, n}^{(1)}\left(x, T ; \sigma, l, \lambda, \varepsilon, \hbar_{m_{k}}\right)\right]^{2}\right\}= \\
=\underline{\lim }_{\substack{\varepsilon \rightarrow 0 \\
\sigma \rightarrow 0}} \lim _{\mathrm{k} \rightarrow \infty} \int d x\left[I_{1}^{(1)}\left(x, T ; \sigma, l, \lambda, \varepsilon, \hbar_{m_{k}}\right)\right]^{2} \leq \\
\leq \lim _{\hbar \rightarrow 0} \int d x\left\{\int_{q(T)=x} D^{+}[q(t)] \Psi(q(0))\left[\left|q_{i}(T)\right|\right]^{\frac{1}{2}} \cos \left[\frac{1}{\hbar} S_{1}(\dot{q}, q, \lambda, T)\right]\right\}^{2} \cdot(\mathrm{A} .41)
\end{gathered}
$$

The inequality (A.41) completed the proof of the statement (1).

(II)Let us estimate now $n$-dimensional path integral 
International Journal of Recent advances in Physics (IJRAP) Vol.3, No.4, November 2014

$$
\begin{gathered}
I_{1, n}^{(2)}(x, T ; \sigma, l, \lambda, \varepsilon)= \\
\int_{q(T)=x} D_{n}^{+}[q(t)] \Psi(q(0))\left[\left|q_{i}(T)\right|\right]^{1 / 2} \exp \left[-\frac{1}{\hbar} \boldsymbol{S}(q, T ; \sigma, l)\right] \sin \left[\frac{1}{\hbar} \boldsymbol{S}_{\varepsilon, 1}(\dot{q}, q, \lambda, T)\right] \sin \left[-\frac{1}{\hbar} \boldsymbol{S}_{\varepsilon, 2}(q, \lambda, T)\right] .(\mathrm{A} .4
\end{gathered}
$$

From Eq. (A.42) one obtain the inequality

$$
\begin{aligned}
& \left|I_{1, n}^{(2)}(x, T ; \sigma, l, \lambda, \varepsilon)\right| \leq \\
& \leq \int_{q(T)=x} D_{n}^{+}[q(t)] \Psi(q(0))\left[\left|q_{i}(T)\right|\right]^{1 / 2} \exp \left[-\frac{1}{\hbar} \boldsymbol{S}(q, T ; \sigma, l)\right]\left|\sin \left[-\frac{1}{\hbar} \boldsymbol{S}_{\varepsilon, 2}(q, \lambda, T)\right]\right| \leq \\
& \leq \sum_{i=0}^{\infty} \frac{\hbar^{-(2 i+1)}}{(2 i+1) !} \int_{q(T)=x} D_{n}^{+}[q(t)] \Psi(q(0))\left[\left|q_{i}(T)\right|\right]^{\frac{1}{2}}\left[\left|\boldsymbol{S}_{\varepsilon, 2}(q, \lambda, T)\right|\right]^{(2 i+1)} \exp \left[-\frac{1}{\hbar} \boldsymbol{S}(q, T ; \sigma, l)\right]= \\
& =\sum_{i=0}^{\infty} \frac{\hbar^{-(2 i+1)}}{(2 i+1) !} \wp_{\varepsilon}^{(i)}(x, T ; \sigma, l, n)(\mathrm{A} .43) \\
& \text { where } \\
& \wp_{\varepsilon}^{(i)}(x, T ; \sigma, l, n)=\int_{q(T)=x} D_{n}^{+}[q(t)] \Psi(q(0))\left[\left|q_{i}(T)\right|\right]^{\frac{1}{2}}\left[\left|\boldsymbol{S}_{\varepsilon, 2}(q, \lambda, T)\right|\right]^{(2 i+1)} \exp \left[-\frac{1}{\hbar} \boldsymbol{S}(q, T ; \sigma, l)\right] .(A \\
& \text { Using replacement } q_{i}(t):=\hbar^{\frac{1}{2}} q_{i}(t), t \in[0, T], i=1, \ldots, d \text { into RHS of the Eq.(A.44) one obtain } \\
& \begin{array}{c}
\wp_{\varepsilon}^{(i)}(x, T ; \sigma, l, n)= \\
\hbar^{1 / 4} \int_{q(T)=\frac{x}{\sqrt{\hbar}}} \breve{D}_{n}^{+}[q(t)] \Psi\left(\hbar^{\frac{1}{2}} q(0)\right)\left[\left|q_{i}(T)\right|\right]^{\frac{1}{2}}\left[\left|\boldsymbol{S}_{\varepsilon, 2}(q, \lambda, T)\right|\right]^{(2 i+1)} \exp \left[-\frac{1}{\hbar} \boldsymbol{S}\left(\hbar^{1 / 2} q, T ; \sigma, l\right)\right]=
\end{array} \\
& \hbar^{1 / 4} \hbar^{i / 2} \int d y \int_{\substack{q(T)=\frac{x}{\sqrt{\hbar}} \\
q(0)=\frac{y}{\sqrt{\hbar}}}} \breve{D}_{n}^{+}[q(t)] \breve{\Psi}(q(0))\left[\left|q_{i}(T)\right|\right]^{\frac{1}{2}}\left[\left|\widehat{\boldsymbol{S}}_{\varepsilon, 2}(q, \lambda, T)\right|\right]^{(2 i+1)} \exp [-\boldsymbol{S}(q, T ; \sigma, l)]= \\
& =\hbar^{1 / 4} \hbar^{i / 2} \widehat{\wp}_{\varepsilon}^{(i)}(x, T ; \sigma, l, n) \text {, where(A.45) } \\
& \breve{D}_{n}^{+}[q(t)]=D_{n}^{+}\left[\hbar^{\frac{1}{2}} q(t)\right], t \in[0, T], \breve{\Psi}(q(0))=\frac{\eta^{d / 4}}{(2 \pi)^{d / 4} \hbar^{d / 4}} \exp \left[\frac{\eta q^{2}(0)}{2}\right] \text {,see Eq. (3.1) and } \\
& \widehat{\boldsymbol{S}}_{\varepsilon, 2}(q, \lambda, T, \hbar)=\int_{0}^{T} \widehat{V}_{\varepsilon, 1}(q(t), t, \lambda, \hbar) d t,
\end{aligned}
$$


International Journal of Recent advances in Physics (IJRAP) Vol.3, No.4, November 2014

$\widehat{V}_{\varepsilon, 1}(q(t), t, \lambda, \hbar)=a_{\varepsilon, 3}(q(t), t, \lambda) q^{3}(t)+\cdots+\hbar^{\frac{\alpha-3}{2}} a_{\varepsilon, \alpha}(q(t), t, \lambda) q^{\alpha}(t)$. (A. 47$)$

$\widehat{\wp}_{\varepsilon}^{(i)}(x, T ; \sigma, l, n)=$

$\int d y \int_{q(T)=\frac{x}{\sqrt{\hbar}}} \breve{D}_{n}^{+}[q(t)] \breve{\Psi}(q(0))\left[\left|q_{i}(T)\right|\right]^{\frac{1}{2}}\left[\left|\widehat{\boldsymbol{S}}_{\varepsilon, 2}(q, \lambda, T)\right|\right]^{(2 i+1)} \exp [-\boldsymbol{S}(q, T ; \sigma, l)] .(\mathrm{A} .48)$ $q(0)=\frac{y}{\sqrt{\hbar}}$

From (A.43)-(A.48) one obtain

$\left|I_{1, n}^{(2)}(x, T ; \sigma, l, \lambda, \varepsilon)\right| \leq \hbar^{\frac{1}{4}} \sum_{i=0}^{\infty} \frac{\hbar^{2(i+1)}}{(2 i+1) !} \widehat{\wp}_{\varepsilon}^{(i)}(x, T ; \sigma, l, n)=\boldsymbol{\Theta}_{\varepsilon}(x, T ; \sigma, l, \hbar, n) .($ A.49)

Let us to choose an sequence $\left\{\hbar_{m}\right\}_{m=1}^{\infty}$ such that

(i) $\quad \lim _{m \rightarrow \infty} \hbar_{m}=0$ and

(ii) $\quad \lim _{\mathrm{m}, \mathrm{n} \rightarrow \infty} \int d x\left\{\boldsymbol{\Theta}_{\varepsilon}^{(m)}\left(x, T ; \sigma, l, \hbar_{m}, n\right)\right\}^{2}=\lim _{m, n \rightarrow \infty} \int d x\left\{\sum_{i=0}^{m} \frac{\hbar_{m}^{2(i+1)}}{(2 i+1) !} \widehat{\wp}_{\varepsilon}^{(i)}(x, T ; \sigma, l, n)\right\}^{2}=0$.

We note that from (ii) follows that: perturbative expansion

$$
\int d x\left\{\boldsymbol{\Theta}_{\varepsilon}\left(x, T ; \sigma, l, \hbar_{m}, n\right)\right\}^{2}=\hbar_{m}^{1 / 4} \int d x\left\{\sum_{i=0}^{\infty} \frac{\hbar^{2(i+1)}}{(2 i+1) !} \widehat{\S}_{\varepsilon}^{(i)}(x, T ; \sigma, l, n)\right\}^{2}
$$

vanishes in the limit $m, n \rightarrow \infty$.From (A.49) one obtain

$\underline{\lim }_{m, n \rightarrow \infty} \int d x\left[I_{1, n}^{(2)}\left(x, T ; \sigma, l, \lambda, \varepsilon, \hbar_{m}\right)\right]^{2} \leq \underline{\lim }_{\mathrm{m}, \mathrm{n} \rightarrow \infty} \int d x\left\{\boldsymbol{\Theta}_{\varepsilon}\left(x, T ; \sigma, l, \hbar_{m}, n\right)\right\}^{2}$. (A.50)

Let us to choose now an subsequence $\left\{\hbar_{m_{k}}\right\}_{m_{k}=1}^{\infty}$ such that the limit:

$\lim _{k, n \rightarrow \infty} \int d x\left[I_{1, n}^{(2)}\left(x, T ; \sigma, l, \lambda, \varepsilon, \hbar_{m_{k}}\right)\right]^{2}$ exist and $\lim _{\mathrm{k}, \mathrm{n} \rightarrow \infty} \int d x\left[I_{1, n}^{(2)}\left(x, T ; \sigma, l, \lambda, \varepsilon, \hbar_{m_{k}}\right)\right]^{2}=$

$\varliminf_{m, n \rightarrow \infty} \int d x\left[I_{1, n}^{(2)}\left(x, T ; \sigma, l, \lambda, \varepsilon, \hbar_{m}\right)\right]^{2}$

From (A.51) and Proposition A.1 one obtain

$\lim _{\mathrm{k}, \mathrm{n} \rightarrow \infty} \int d x\left[I_{1, n}^{(2)}\left(x, T ; \sigma, l, \lambda, \varepsilon, \hbar_{m_{k}}\right)\right]^{2}=\lim _{k \rightarrow \infty}\left\{\lim \mathrm{n} \rightarrow \infty \int d x\left[I_{1, n}^{(2)}\left(x, T ; \sigma, l, \lambda, \varepsilon, \hbar_{m_{k}}\right)\right]^{2}\right\}$.

From (A.50), (A.51) and (A.52) one obtain

$$
\begin{gathered}
\underline{\lim }_{\substack{\varepsilon \rightarrow 0 \\
\sigma \rightarrow 0}} \underline{\lim }_{\hbar \rightarrow 0} \int d x\left[I_{1}^{(2)}(x, T ; \sigma, l, \lambda, \varepsilon)\right]^{2} \leq \\
\leq \underline{\lim }_{\substack{\varepsilon \rightarrow 0 \\
\sigma \rightarrow 0}} \lim _{\mathrm{k} \rightarrow \infty}\left\{\lim \mathrm{n} \rightarrow \infty \int d x\left[I_{1, n}^{(2)}\left(x, T ; \sigma, l, \lambda, \varepsilon, \hbar_{m_{k}}\right)\right]^{2}\right\}= \\
=\underline{\lim }_{\substack{\varepsilon \rightarrow 0 \\
\sigma \rightarrow 0}} \lim _{\mathrm{k} \rightarrow \infty} \int d x\left[I_{1}^{(2)}\left(x, T ; \sigma, l, \lambda, \varepsilon, \hbar_{m_{k}}\right)\right]^{2}=0 .
\end{gathered}
$$


International Journal of Recent advances in Physics (IJRAP) Vol.3, No.4, November 2014

Proof of the statements (3)-(6) is similarly to the proof of the statements (1)-(2).

Theorem A.1.Let $I_{1}(x, T ; \sigma, l, \lambda, \varepsilon, \hbar)=I_{1}(x, T ; \sigma, l, \lambda, \varepsilon), I_{2}(x, T ; \sigma, l, \lambda, \varepsilon, \hbar)=I_{2}(x, T ; \sigma, l, \lambda, \varepsilon)$, where $I_{1}(x, T ; \sigma, l, \lambda, \varepsilon)$ is given via Eq.(A.22a)-Eq.(A.22b). Then

$$
\begin{gathered}
\underline{\lim }_{\substack{\varepsilon \rightarrow 0 \\
\sigma \rightarrow 0}} \underline{\lim _{\hbar \rightarrow 0} \int d x\left[I_{1}^{2}(x, T ; \sigma, l, \lambda, \varepsilon)\right] \leq} \\
\leq \lim _{\hbar \rightarrow 0} \int d x\left\{\int_{q(T)=x} D^{+}[q(t)] \Psi(q(0))\left[\left|q_{i}(T)\right|\right]^{\frac{1}{2}} \cos \left[\frac{1}{\hbar} \boldsymbol{S}_{1}(\dot{q}, q, \lambda, T)\right]\right\}^{2},(\mathrm{~A} .53 . \mathrm{a}) \\
\underline{\lim }_{\sigma \rightarrow 0} \underline{\lim }_{\hbar \rightarrow 0} \int d x\left[I_{2}^{2}(x, T ; \sigma, l, \lambda, \varepsilon)\right] \leq \\
\leq \lim _{\hbar \rightarrow 0} \int d x\left\{\int_{q(T)=x} D^{+}[q(t)] \Psi(q(0))\left[\left|q_{i}(T)\right|\right]^{\frac{1}{2}} \sin \left[\frac{1}{\hbar} S_{1}(\dot{q}, q, \lambda, T)\right]\right\}^{2},
\end{gathered}
$$

Here

$\boldsymbol{S}_{1}(\dot{q}, q, \lambda, T)=\boldsymbol{S}_{\varepsilon=0,1}(\dot{q}, q, \lambda, T)=\int_{0}^{T} L_{\varepsilon=0}(\dot{q}(t), q(t), t, \lambda) d t,(\mathrm{~A} .54)$

$L_{\varepsilon=0}(\dot{q}(t), q(t), t, \lambda)=\frac{m}{2} \dot{q}^{2}(t)-V_{\varepsilon=0,0}(q(t), t, \lambda) .(\mathrm{A} .55)$

Proof. We remain that

$I_{1}(x, T ; \sigma, l, \lambda, \varepsilon, \hbar)=I_{1}^{(1)}(x, T ; \sigma, l, \lambda, \varepsilon, \hbar)+I_{1}^{(2)}(x, T ; \sigma, l, \lambda, \varepsilon, \hbar) .(\mathrm{A} .56)$

From Eq.(A.56) we obtain

$$
\begin{gathered}
\int d x\left[I_{1}^{2}(x, T ; \sigma, l, \lambda, \varepsilon, \hbar)\right] \leq \int d x\left[I_{1}^{(1)}(x, T ; \sigma, l, \lambda, \varepsilon, \hbar)\right]^{2}+ \\
\int d x\left[I_{1}^{(2)}(x, T ; \sigma, l, \lambda, \varepsilon, \hbar)\right]^{2}+2 \int d x\left[\left|I_{1}^{(1)}(x, T ; \sigma, l, \lambda, \varepsilon) I_{1}^{(2)}(x, T ; \sigma, l, \lambda, \varepsilon, \hbar)\right|\right]= \\
=\int d x\left[I_{1}^{(1)}(x, T ; \sigma, l, \lambda, \varepsilon, \hbar)\right]^{2}+\int d x\left[I_{1}^{(2)}(x, T ; \sigma, l, \lambda, \varepsilon, \hbar)\right]^{2}+ \\
+2 \sqrt{\int d x\left[I_{1}^{(1)}(x, T ; \sigma, l, \lambda, \varepsilon, \hbar)\right]^{2} \int d x\left[I_{1}^{(2)}(x, T ; \sigma, l, \lambda, \varepsilon, \hbar)\right]^{2}} .(\mathrm{A} .57)
\end{gathered}
$$

Let us to choose now an sequences $\left\{\hbar_{m}\right\}_{m=1}^{\infty},\left\{\varepsilon_{k}\right\}_{k=1}^{\infty},\left\{\sigma_{l}\right\}_{l=1}^{\infty}$ such that:

$$
\begin{gathered}
\text { (i) } \lim _{m \rightarrow \infty} \hbar_{m}=0, \lim _{k \rightarrow \infty} \varepsilon_{k}=0, \lim _{l \rightarrow \infty} \sigma_{l}=0 \\
\text { (ii) } \lim _{\substack{\mathrm{k} \rightarrow \infty \\
l \rightarrow \infty}} \lim _{\mathrm{m} \rightarrow \infty} \int d x\left[I_{1}^{(2)}\left(x, T ; \sigma_{l}, l, \lambda, \varepsilon_{k}, \hbar_{m}\right)\right]^{2}= \\
=\underline{\lim }_{\substack{k \rightarrow \infty \\
l \rightarrow \infty}} \underline{\lim }_{m \rightarrow \infty} \int d x\left[I_{1}^{(2)}\left(x, T ; \sigma_{l}, l, \lambda, \varepsilon_{k}, \hbar_{m}\right)\right]^{2}=0,(\mathrm{~A} .58)
\end{gathered}
$$


International Journal of Recent advances in Physics (IJRAP) Vol.3, No.4, November 2014

(iii)

$$
\underline{\lim }_{\substack{k \rightarrow \infty \\ l \rightarrow \infty}} \underline{\lim }_{m \rightarrow \infty} \int d x\left[I_{1}^{(1)}\left(x, T ; \sigma_{l}, l, \lambda, \varepsilon_{k}, \hbar_{m}\right)\right]^{2} \leq
$$

$\leq \lim _{\hbar \rightarrow 0} \int d x\left\{\int_{q(T)=x} D^{+}[q(t)] \Psi(q(0))\left[\left|q_{i}(T)\right|\right]^{\frac{1}{2}} \cos \left[\frac{1}{\hbar} \boldsymbol{S}_{1}(\dot{q}, q, \lambda, T)\right]\right\}^{2}$

Therefore from inequality(A.57),Eq.(A.58) and inequality(A.59)we obtain

$$
\begin{aligned}
& \underline{\lim }_{\varepsilon \rightarrow 0} \underline{\lim }_{\hbar \rightarrow 0} \int d x\left[I_{1}^{2}(x, T ; \sigma, l, \lambda, \varepsilon, \hbar)\right] \leq \underline{\lim }_{k \rightarrow \infty} \underline{\lim }_{m \rightarrow \infty} \int d x\left[I_{1}^{2}\left(x, T ; \sigma_{l}, l, \lambda, \varepsilon_{k}, \hbar_{m}\right)\right] \leq \\
& \leq \underline{\lim }_{\substack{\mathrm{k} \rightarrow \infty \\
l \rightarrow \infty}} \underline{\lim }_{m \rightarrow \infty} \int d x\left[I_{1}^{(1)}\left(x, T ; \sigma_{l}, l, \lambda, \varepsilon_{k}, \hbar_{m}\right)\right]^{2}+\lim _{\substack{\mathrm{k} \rightarrow \infty \\
l \rightarrow \infty}} \lim _{\mathrm{m} \rightarrow \infty} \int d x\left[I_{1}^{(2)}\left(, T ; \sigma_{l}, l, \lambda, \varepsilon_{k}, \hbar_{m}\right)\right]^{2}+ \\
& +2 \lim _{\substack{k \rightarrow \infty \\
l \rightarrow \infty}} \lim _{\mathrm{m} \rightarrow \infty} \sqrt{\int d x\left[I_{1}^{(1)}\left(, T ; \sigma_{l}, l, \lambda, \varepsilon_{k}, \hbar_{m}\right)\right]^{2} \int d x\left[I_{1}^{(2)}\left(, T ; \sigma_{l}, l, \lambda, \varepsilon_{k}, \hbar_{m}\right)\right]^{2}}= \\
& \underline{\lim }_{l \rightarrow \infty} \underline{\lim }_{m \rightarrow \infty} \int d x\left[I_{1}^{(1)}\left(x, T ; \sigma_{l}, l, \lambda, \varepsilon_{k}, \hbar_{m}\right)\right]^{2}= \\
& =\lim _{\hbar \rightarrow 0} \int d x\left\{\int_{q(T)=x} D^{+}[q(t)] \Psi(q(0))\left[\left|q_{i}(T)\right|\right]^{\frac{1}{2}} \cos \left[\frac{1}{\hbar} \boldsymbol{S}_{1}(\dot{q}, q, \lambda, T)\right]\right\}^{2} .
\end{aligned}
$$

\section{References}

[1] Vijay, R., Slichter, D. H. \&Siddiqi, I. Observation of quantum jumps in a superconducting artificial atom. Phys. Rev. Lett.106, 110502 (2011)

[2] Peil, S. \&Gabrielse, G. Observing the quantum limit of an electron cyclotron: QND measurements of quantum jumps between Fock states. Phys. Rev. Lett.83, 1287-1290 (1999)

Nagourney, W., Sandberg, J. \&Dehmelt, H. Shelved optical electron amplifier: observation of quantum jumps. Phys. Rev. Lett.56, 2797-2799 (1986)

[3] Sauter, T., Neuhauser, W., Blatt, R. \&Toschek, P. E. Observation of quantum jumps.Phys. Rev. Lett.57,1696-1698 (1986)

[4] Bergquist, J. C., Hulet, R. G., Itano, W. M. \&Wineland, D. J. Observation of quantum jumps in a single atom. Phys. Rev. Lett.57, 1699-1702 (1986)

[5] N. Bohr, Philos. Mag. 26, 1,1913.

[6] R. Dum, P. Zoller, and H. Ritsch, Phys. Rev. A 45, 4879, 1992.

[7] J. Dalibard, Y. Castin, and K. Mølmer, Phys. Rev. Lett. 68, 5801992.

[8] DariuszGatarek,Continuous quantum jumps and infinite-dimensional stochastic equations, J. Math. Phys. 32 (8), August 1991

[9] K. Molmer, Y. Castin, and J. Dalibard, J. Opt. Soc. Am. B 10,524,1993.

[10] C. W. Gardiner, A. S. Parkins, and P. Zoller, Wave-function quantum stochastic differential equations and quantum-jump simulation methods,Phys. Rev. A 46, 4363 - Published 1 October 1992

[11] Nicolas Vogt, Jan Jeske, and Jared H. Cole,Stochastic Bloch-Redfield theory: Quantum jumps in a solid-stateenvironment,Phys. Rev. B 88, 174514 - Published 19 November 2013

[12] J. E. Reiner,H. M. Wiseman,H. Mabuchi,Quantum jumps between dressed states: A proposed cavity-QED test using feedback,PHYSICAL REVIEW A 67, 042106 (2003)

[13] D. J. Berkeland,_ D. A. Raymondson, and V. M. Tassin, Tests for non-randomness in quantum jumps.Phys. Rev. A 69, 052103 - Published 7 May 2004

[14] H. M. Wiseman and G. J. Milburn, Phys. Rev. A 47, 1652, 1993.

[15] H. M. Wiseman and G. E. Toombes, Phys. Rev. A 60, 2474,1999.

[16] M.Stojanovic,Regularizationforheat kernel innonlinearparabolicequations,TAIWANESE JOURNAL 
International Journal of Recent advances in Physics (IJRAP) Vol.3, No.4, November 2014

OF MATHEMATICSVol. 12, No. 1, pp. 63-87, February 2008 This paper is available online athttp://www.tjm.nsysu.edu.tw/

[17] C. Garetto,Fundamental Solutions in the ColombeauFramework:Applications to Solvability and Regularity Theory,ActaAppl Math (2008) 102: 281-318.DOI 10.1007/s10440-008-9220-8

[18] J. Foukzon, A. A. Potapov, S. A. Podosenov, Exact quasiclassicalasymptotics beyond Maslov canonical operator. http://arxiv.org/abs/1110.0098Podosenov SA, Potapov AA, J. Foukzon., Menkova ER, 'Nonholonomic, fractal and related structures in the relativistic continuous media, electrodynamics, quantum mechanics and cosmology: Asymptotic methods in problems of classical and fractal pulse electrodynamics.'2015 paperback.In press.

ISBN 978-5-9710-1525-3

[19] E.Nelson,Feynman integrals and the Schrödinger equation,J.Math.Phys.5(1964), pp.332--343.

[20] V.P.Maslov,Complex Markov Cheins and Continual FeynmanIntegral.NaukaMoskov 1976.

[21] T. M. Apostol, Mathematical Analysis, Second edition, Addison Wesley,1974.

[22] W. W. L. Chen, Fundamentals of Analysis, Published by W. W. L. Chen viaInternet, 2003.

[23] S. L. Gupta and N. Rani, Principles of Real Analysis, VikasPuplishingHouse, New Delhi, 1998.

[24] J. Lehmann, P.Reimann, and P.Hanggi,Surmounting Oscillating Barriers: Path-integral approach for Weak Noise,Phys. Rev. E 62, 6282 (2000)

[25] M.V. Fedoryuk, "The method of steepest descent", Moscow (1977) (In Russian),

[26] M.V. Fedoryuk, "Asymptotic Methods in Analysis",Encyclopaedia of Mathematical Sciences, Volume 13, 1989, pp 83-191.

[27] Z. Shun and P.McCullagh, "Laplace Approximation of High Dimensional Integrals,"Journal of the Royal Statistical Society. Series B(Methodological)Vol. 57, No. 4 (1995), pp. 749-760

[28] J. Foukzon,Strong Large Deviations Principles of Non-Freidlin-Wentzell Type - Optimal Control Problem with Imperfect Information - Jumps Phenomena in Financial Markets,Communications in Applied Sciences, Volume 2, Number 2, 2014, 230-363.ISSN 2201-7372 